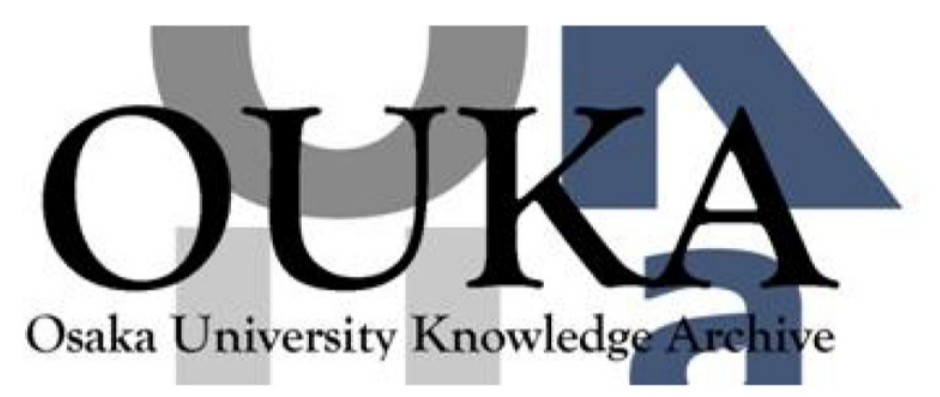

\begin{tabular}{|c|l|}
\hline Title & $\begin{array}{l}\text { Interaction problem between fluid and membrane } \\
\text { by a consistent direct discretisation approach }\end{array}$ \\
\hline Author(s) & $\begin{array}{l}\text { Takeuchi, Shintaro; Fukuoka, Hiroki; Gu, } \\
\text { Jingchen et al. }\end{array}$ \\
\hline Citation & $\begin{array}{l}\text { Journal of Computational Physics. 371 p. 1018- } \\
\text { p. 1042 }\end{array}$ \\
\hline Issue Date & $2018-10-15$ \\
\hline oaire:version & AM \\
\hline URL & https://hdl. handle. net/11094/77254 \\
\hline rights & $\begin{array}{l}\text { ○ 2018. This manuscript version is made } \\
\text { avai lable under the CC-BY-NC-ND 4.0 License }\end{array}$ \\
\hline Note & \\
\hline
\end{tabular}

Osaka University Knowledge Archive : OUKA

https://ir. Library. osaka-u. ac. jp/

Osaka University 


\title{
Interaction problem between fluid and membrane by a consistent direct discretisation approach
}

\author{
Shintaro Takeuchi ${ }^{1, *}$, Hiroki Fukuoka ${ }^{1}$ Jingchen $\mathbf{G u}^{1}$ and Takeo Kajishima ${ }^{1}$ \\ ${ }^{1}$ Department of Mechanical Engineering, Osaka University \\ 2-1 Yamada-oka, Suita-city, Osaka 565-0871 Japan \\ * Corresponding author, E-mail: shintaro.takeuchi@mech.eng.osaka-u.ac.jp
}

\begin{abstract}
Numerical methods for solving the flow around a moving or deforming membrane are developed by employing the ideas of the direct-discretisation of the Navier-Stokes equation even in the immediate vicinity of the object surface and consistent-discretisation of the pressure Poisson equation with the incompressible velocity field (i.e., consistent-coupling) that incorporates the boundary conditions on the surface to the pressure equation. Comparative study of three different discretisation strategies is carried out to highlight the effect of the consistent and direct discretisations. The validity of the present "consistent direct discretisation" is established through comparisons with the analytical solutions for some fundamental shear-driven 2-D flow fields with a moving membrane of a flat and corrugated geometries. Also, the flows induced by a membrane of circular geometry (i.e., particle) are compared with the reference numerical results under prescribed motion in a stationary fluid as well as lateral migration in a shear flow. A three dimensional problem is presented for an interaction problem between a spherical particle and Taylor-Green vortices, and the result is verified by comparing with the independently-conducted numerical results. Finally, the method is applied to an interaction problem between the fluid and flexible filament showing strong flapping and snapping motions.
\end{abstract}

\section{Key words:}

Discrete-forcing; Immersed boundary; Membrane; Moving boundary; Particle 


\section{Introduction}

The interaction between a fluid and solid object is observed in a variety of problems in industrial applications and biological flows, such as the flows including fibres, solid particles, capsules and biological cells. The modelling of the fluid-object interaction, particularly fluid-mediated multiple-body interaction, is understood as one of the challenging subjects for computational fluid mechanics. For those problems, a fixed-mesh approach is often employed regardless of the object geometry, and the fluid motion is solved based on an Eulerian frame. The fluid-object interface is segmented by the fluid mesh, and each segment immersed in the fluid experiences the fluid force. On the other hand, the effect of the object is incorporated into the fluid governing equation or discretised equation as an external force.

In the immersed boundary methods of continuous forcing type, pioneered by Peskin [1] and further developed by Goldstein et al. [2] and other researchers, the object geometry is represented by connected Lagrangian markers, and the fluid force is projected onto the marker position by a continuous function model of the delta function, and the same function is used to distribute the nodal force onto the Eulerian field. Another popular method of fixed-mesh approach is discrete-forcing method (also known as directforcing method), originally developed by Mohd-Yusof [3] for three-dimensional immersed object in a fluid. In this method, the interaction force for imposing the no-slip condition is directly incorporated into the discretised equation. Later, Fadlun et al. [4] applied the discrete-forcing idea for a moving object. Kim et al. [5] proposed an improved idea of the discrete-forcing method by adjusting the mass conservation at the interface. However, because the object surface generally does not align with the face of the computational cell, it is hard to correctly calculate the hydrodynamic force associated with the no-slip and pressure boundary conditions at the object surface. The existing methods experience the above problem for imposing the boundary conditions at the interface, particularly for moving boundary problems, causing deterioration in numerical accuracy due to the treatment of the flow field near the object surface.

For example, in the methods employing a regularised-delta function, because the nodal information (velocity and force on the interface) is distributed over the fluid cells in the vicinity of the interface, the interface has a substantial thickness, which decreases the accuracy of the fluid motion near the interface. Meanwhile, for the discrete-forcing approaches that commonly resolve the interface as a sharp edge, Ikeno and Kajishima [6;7] pointed out an inconsistency between the incompressible velocity and pressure fields. They proposed a proper discretisation procedure for the pressure Poisson equation that guarantees the velocitypressure consistency near the object surface. Dependence of the numerical result on the time increment is also often pointed out to be a critical reason for the reduction in accuracy. Sato et al. [8] proposed a method that addresses those problems by directly discretising the governing equations even in the immediate vicinity of the interface. By employing a collocated Cartesian grid system, they treat the primary variables (such as velocity, pressure and temperature) at the cell centres by directly solving the governing equations with the consistent discretisation for the pressure Poisson equation, while the secondary properties (such as derivatives and cell-face velocities) are obtained by interpolation and extrapolation [8]. The consistency 
is guaranteed by considering the distance from the cell centre to the interface and by carefully designing the discretisation stencils with the primary and secondary variables. They mentioned that, in the vicinity of the immersed boundary, both solving the governing equations for the primary variables and undertaking the velocity-pressure consistency are equally essential for accurately satisfying the mass and momentum conservations under given boundary conditions associated with the interface. Their "consistent direct discretisation" approach was found to substantially improve the numerical accuracy for the flow field around a stationary solid object of a finite-volume, and the method showed that the numerical accuracy is insensitive to the Courant numbers (under a fixed spatial resolution) [8].

Another problem involving an immersed boundary is spurious pressure oscillation due to moving boundary. Breugem [9] mentioned that, for fixed-mesh approaches, the high-frequency oscillations in the force and torque are caused by the violation of the translational invariance in the interpolation operation. Seo and Mittal [10] demonstrated the pressure oscillations associated with the appearance of the fresh and dead cells (as the spurious mass source and sink, respectively) and mentioned the significance of the geometric conservation law for reducing the pressure oscillations. They employed a cut-cell method to mass conservation in a problem of an oscillating cylinder, and successfully reduced the pressure spikes. The above two studies suggest that the treatment of the discontinuous change of the stencils due to the passing interface is of importance for fixed-mesh approaches in general, and therefore, the methods with sharp edge treatment like the discrete-forcing approach particularly needs more attention to the consistency in velocity-pressure coupling for ensuring the mass and momentum conservations in a moving boundary problem.

In the present work, moving boundary treatment in the fixed-mesh approach is further explored by undertaking the consistency in the velocity-pressure coupling and the direct discretisation of the momentum equation even in the immediate vicinity of the moving interface. Although the consistent direct discretisation approach [8] has been constructed to cover a case with a moving boundary, the method does not account for the discrete change of the discretisation stencils due to the passing interface. This problem particularly exacerbates in the case with a thin object of virtually zero-thickness. In the present study, a solution approach for moving/deforming boundary treatment of the consistent direct discretisation is presented for thin object by taking account of the front and rear sides of the moving membrane for determining the discretisation stencils in the vicinity of the interface. Through comparisons with analytical solutions for some fundamental flow fields with a moving membrane, the validity of the present moving boundary treatment for the consistent direct discretisation is established. Time histories of the hydrodynamic force on an object and trajectories are investigated for externally-oscillated circular membrane and migrating (rigid/capsule) particle in a wall-bounded shear flow. For a 3-D problem, an interaction between a spherical particle and a vortical flow is conducted. The results are compared with the reference numerical data in the literature to show that the proposed consistent direct discretisation is a fundamental tool to fluid-particle interaction problem on a Cartesian system. The applicability to an interaction problem between a flexible membrane and a uniform flow is also demonstrated. 


\section{Governing equations for fluid and membrane}

Throughout the present paper, the material properties, such as density, viscosity and bending stiffness, are assumed to be constant. The governing equations are non-dimensionalised based on the reference length $L$, reference velocity $U$ and the reference pressure $\rho_{f} U^{2}$, where $\rho_{f}$ is the fluid density. The following three characteristic non-dimensional numbers exist: the Reynolds number $\operatorname{Re}=U L / \nu$, the density ratio of the membrane to the fluid $r=\rho_{s} b / \rho_{f} L$ [11] and the non-dimensional bending stiffness $\gamma=E I / \rho_{f} U^{2} L^{3}$, where $\nu$ is the kinetic viscosity, $\rho_{s} b$ the mass per unit length of the membrane with a characteristic scale of the membrane $b$ and $E I$ is the bending rigidity.

The governing equations for incompressible flow are the continuity equation and Navier-Stokes (N-S) equation:

$$
\begin{aligned}
& \nabla \cdot \boldsymbol{u}=0 \\
& \frac{\partial \boldsymbol{u}}{\partial t}+\boldsymbol{u} \cdot \nabla \boldsymbol{u}=\nabla \cdot \boldsymbol{\sigma}+\boldsymbol{f}_{\text {ext }}
\end{aligned}
$$

where $\boldsymbol{u}\left(=(u, v)\right.$ or $\left(u_{1}, u_{2}\right)$ in the Cartesian coordinate system) is the velocity, $t$ the time and $\boldsymbol{f}_{\text {ext }}$ the external force. The stress tensor $\boldsymbol{\sigma}$ for a Newtonian fluid is given by the following form:

$$
\boldsymbol{\sigma}=-p \boldsymbol{I}+\frac{1}{\operatorname{Re}}\left(\nabla \boldsymbol{u}+(\nabla \boldsymbol{u})^{T}\right)
$$

where $p$ is the pressure and $\boldsymbol{I}$ is the identity tensor.

For solid object, a membrane of open/closed geometry under prescribed/free motion in a fluid is employed in two dimensions, unless specified otherwise. For non-deformable object, Newton's equation of motion is solved for translating and angular velocities. For flexible membrane, the governing equation is as follows:

$$
r \frac{\partial^{2} \boldsymbol{X}}{\partial t^{2}}=\frac{\partial}{\partial s}\left(T \frac{\partial \boldsymbol{X}}{\partial s}\right)+\boldsymbol{F}+\boldsymbol{B}
$$

where $\boldsymbol{X}$ is the location of material point on the membrane, $s$ the arc length along the surface, $T$ the tension, $\boldsymbol{F}$ the force exerted by the fluid and $\boldsymbol{B}$ the bending force determined by:

$$
\boldsymbol{B}=-\frac{\partial^{2}}{\partial s^{2}}\left(\gamma \frac{\partial^{2} \boldsymbol{X}}{\partial s^{2}}\right)
$$

In the present study, the membrane is assumed to be infinitely thin and inextensible. The inextensible condition is represented as follows:

$$
\frac{\partial \boldsymbol{X}}{\partial s} \cdot \frac{\partial \boldsymbol{X}}{\partial s}=1
$$

Deformation of membrane is only relevant to Section 5, and, in the other sections, the membrane geometry is fixed and/or the motion is prescribed in a flow. 


\section{Numerical method}

The fluid and object are alternatively time-updated. For each time step, the membrane velocity and position are updated, followed by the time-marching procedure of the fluid phase.

In the following numerical procedure, the fluid force on the Eulerian frame is projected onto the membrane, and the effect of the presence of the membrane on the fluid is incorporated as the boundary condition.

\subsection{Time update of the fluid}

The fluid equations are spatially discretised by a second-order central finite difference on a collocated grid, and a fractional step method is employed for the velocity-pressure coupling. For the time-marching of the N-S equation, unless specified otherwise, the second-order Adams-Bashforth and the Crank-Nicolson methods are employed for the convective and viscous terms, respectively.

The procedure for updating the fluid phase is explained in the following. Hereafter, $\delta / \delta x_{k}$ stands for a second-order central finite difference operator for the first-order derivative in the $x_{k}$ direction and the superscript $n$ represents the discrete time level.

1. The N-S equation is time advanced with the pressure gradient term and implicit treatment of the viscous term [12] to obtain the predicted velocity $u_{k}^{\mathrm{p}}(k=1,2)$ :

$$
\frac{u_{k}^{\mathrm{p}}-u_{k}^{n}}{\Delta t}=-\frac{\delta p^{n}}{\delta x_{k}}+\frac{3 C_{k}^{n}-C_{k}^{n-1}}{2}+\frac{1}{\operatorname{Re}} \frac{\delta}{\delta x_{l}} \frac{\delta}{\delta x_{l}}\left(\frac{u_{k}^{\mathrm{p}}+u_{k}^{n}}{2}\right),
$$

where $C_{k}^{n}$ represents the convective term of the conservative form, $\left(\nabla \cdot(\boldsymbol{u} \otimes \boldsymbol{u}) \cdot \boldsymbol{e}_{k}\right)^{n}$

2. The velocity $u_{k}^{* *}$ is calculated by removing the effect of the pressure gradient from $u_{k}^{\mathrm{p}}$.

$$
u_{k}^{* *}=u_{k}^{\mathrm{p}}+\Delta t \frac{\delta p^{n}}{\delta x_{k}} .
$$

3. The cell-face (i.e., the surface of the control volume) velocity $U_{k}^{* *}(k=1,2)$ is constructed from the cell-centred velocity $u_{k}^{* *}$ by interpolation:

$$
U_{k}^{* *}={\overline{u_{k}^{* *}}}^{x_{k}}
$$

where ${\overline{(\cdot)^{x}}}^{x_{k}}$ stands for a second-order interpolation with respect to $x_{k}$. No summation convention applies for the repeating indices in the right-hand side of the above equation.

4. The pressure Poisson equation is solved to obtain the pressure $p$ at the next time level:

$$
\frac{\delta}{\delta x_{l}}\left(\frac{\delta p^{n+1}}{\delta x_{l}}\right)=\frac{1}{\Delta t} \frac{\delta U_{k}^{* *}}{\delta x_{k}} .
$$


5. The cell-face and cell-centred velocities are corrected by the pressure field:

$$
\begin{aligned}
& U_{k}^{n+1}=U_{k}^{* *}-\Delta t \frac{\delta p^{n+1}}{\delta x_{k}}, \\
& u_{k}^{n+1}=u_{k}^{* *}-\Delta t \frac{\delta p^{n+1}}{\delta x_{k}} .
\end{aligned}
$$

Hereafter, the Cartesian components $\left(U_{1}, U_{2}\right),\left(u_{1}^{\mathrm{p}}, u_{2}^{\mathrm{p}}\right)$ are also denoted as $(U, V),\left(u^{\mathrm{p}}, v^{\mathrm{p}}\right)$, respectively, to specify the cell indices.

\subsection{Forcing methods for the fluid phase}

A membrane immersed in a fluid is set up in two dimensions, as shown in Fig. 1. The effect of the fluidmembrane interface is incorporated into the Eulerian equation of the fluid with a set of boundary conditions specified on the membrane surface.

In the following, three different discretisation strategies are introduced. Fundamental idea of the discretisation procedure is briefly reviewed as Case 1 , followed by the consistent direct discretisation procedure based on the method by Sato et al. [8] and its improved idea, as Cases 2 and 3.

Hereafter, the computational cells partitioned by the object boundary are referred to as "boundary cells", as represented by triangles in Fig. 1, which are typically characterised by the segmented centre-to-centre lines (connecting the adjacent cell centres shown by the broken lines) by the membrane.

\subsubsection{Case 1: Discrete forcing approach based on Ref. [4]}

By following the standard treatment of the discrete-forcing method [4], the boundary condition is enforced by specifying the fluid velocity in the boundary cells through interpolations from the velocities on the solid surface and the predicted fluid velocity in the neighbouring cell. In Fig. 1, the $x$ component of the velocity $\left(u^{\mathrm{p}}\right)$ on the boundary cell $(i, j)$ is predicted by the linear interpolation function $\phi_{1}$ :

$$
\begin{aligned}
u_{i, j}^{\mathrm{p}} & =\left[\phi_{1}\left(u^{\mathrm{p}}\right)\right]_{i, j} \\
& =\frac{1}{\epsilon_{x}+1}\left(\epsilon_{x} u_{i+1, j}^{\mathrm{p}}+u_{\mathrm{b}}^{n+1}\right) \quad\left(0<\epsilon_{x}<1\right) .
\end{aligned}
$$

Here, $\epsilon_{x}$ is the non-dimensional distance in the $x$ direction from the cell centre $(i, j)$ to the object surface normalised by the grid spacing $\Delta x$, and $u_{\mathrm{b}}$ is the $x$ component of the fluid velocity defined on the object boundary at the point represented by the cross symbol $(\times)$ in Fig. 1. Due to the no-slip condition, $u_{\mathrm{b}}$ is the same as the membrane velocity. On the same boundary cell, the interpolation of the $y$ component of the velocity $\left(v_{i, j}^{\mathrm{p}}\right)$ is given as follows:

$$
v_{i, j}^{\mathrm{p}}=\frac{1}{\epsilon_{x}+1}\left(\epsilon_{x} v_{i+1, j}^{\mathrm{p}}+v_{\mathrm{b}}^{n+1}\right),
$$

where $v_{\mathrm{b}}$ is the $y$ component of the fluid velocity on the boundary defined at the same point as $u_{\mathrm{b}}$. 
Note that the cells of indices $(i-1, j+1)$ and $(i-1, j)$ in Fig. 1 are partitioned by the interface in the two directions: for $(i-1, j)$ cell, the interface cuts through the two centre-to-centre lines of $(i-1, j)$ $(i-1, j+1)$ and $(i-1, j)-(i, j)$. Also, for the configuration in Fig. 2, the cell $(i, j)$ is partitioned by the interface in the two directions. For those cells, the velocity interpolation formula, Eq. (13), is applied in both directions, and the average of those is assigned as the value at the bidirectionally-opened boundary cell; for example, for the configuration in Fig. 2,

$$
u_{i, j}^{\mathrm{p}}=\frac{1}{2}\left[\frac{1}{\epsilon_{x}+1}\left(\epsilon_{x} u_{i+1, j}^{\mathrm{p}}+\left[u_{\mathrm{b}}\right]_{(i, j)}^{(i-1, j)}\right)+\frac{1}{\epsilon_{y}+1}\left(\epsilon_{y} u_{i, j+1}^{\mathrm{p}}+\left[u_{\mathrm{b}}\right]_{(i, j-1)}^{(i, j)}\right)\right],
$$

where the time level for $u_{\mathrm{b}}$ is omitted for simplicity. Instead, the two cell indices attached to $u_{\mathrm{b}}$ indicate the centre-to-centre line which defines the boundary velocity $u_{\mathrm{b}}$ at the intersection with the interface. At every iteration in the Crank-Nicolson method, the predicted velocity at the boundary cell is given by the above interpolations.

For the configuration of Fig. 1, the velocity $U^{* *}$ on the cell face, $(i-1 / 2, j)$, is given by the interpolation function $\phi_{2}$ from the velocities of the object surface and the fluid on the cell faces:

$$
\begin{aligned}
U_{i-\frac{1}{2}, j}^{* *} & =\left[\phi_{2}\left(U^{* *}\right)\right]_{i-\frac{1}{2}, j} \\
& =\frac{1}{\epsilon_{x}+0.5}\left(\left(\epsilon_{x}-0.5\right) U_{i+\frac{1}{2}, j}^{* *}+u_{\mathrm{b}}^{n+1}\right) .
\end{aligned}
$$

In this strategy of Case 1, by following the discrete-forcing idea of Fadlun et al. [4], the above discretisation schemes, Eqs. (13) and (14), are applied to those terms in the N-S equation (Eq. (7)), the right-hand side of the pressure Poisson equation (Eq. (10)) and the velocity corrections (Eqs. (11) and (12)). The pressure Poisson equation is discretised by a central finite difference scheme, regardless of the presence of the object. Therefore, the coefficient matrix for the Poisson equation is symmetric. The boundary treatment results in the violation of the no-slip boundary condition on the membrane surface, as the pressure boundary treatment is not consistent with that for the velocity field [8].

A special treatment for the discrete change of the stencil is necessary when the membrane jumps over a cell centre. This is a common issue for the discrete-forcing methods, including the following Cases 2 and 3 , and the detail will be explained later. 


\subsubsection{Case 2: Consistent direct discretisation based on Ref. [8]}

In this case, the N-S equation is discretised by taking account of the distances to the interface from the centre of the boundary cell, so that the mass and momentum in the boundary cell are accurately conserved. Based on the configuration in Fig. 1, the discretisations of the $x$ component of the $\mathrm{N}-\mathrm{S}$ equation at the boundary cell $(i, j)$ is explained in the following.

- Convective term:

$$
\begin{aligned}
{\left[\frac{\partial(U u)}{\partial x}+\frac{\partial(V u)}{\partial y}\right]_{i, j}=} & \frac{1}{\Delta x}\left(U_{i+\frac{1}{2}, j} \frac{u_{i, j}+u_{i+1, j}}{2}-U_{i-\frac{1}{2}, j}\left[\phi_{3}(u)\right]_{i-\frac{1}{2}, j}\right) \\
& +\frac{1}{\Delta y}\left(V_{i, j+\frac{1}{2}} \frac{u_{i, j}+u_{i, j+1}}{2}-V_{i, j-\frac{1}{2}} \frac{u_{i, j}+u_{i, j-1}}{2}\right),
\end{aligned}
$$

where

$$
\left[\phi_{3}(u)\right]_{i-\frac{1}{2}, j}=\frac{\left(\epsilon_{x}-0.5\right) u_{i, j}+0.5 u_{\mathrm{b}}^{n+1}}{\epsilon_{x}}
$$

- Viscous term:

$$
\begin{array}{r}
{\left[\frac{\partial^{2} u}{\partial x^{2}}+\frac{\partial^{2} u}{\partial y^{2}}\right]_{i, j}=\frac{1}{0.5\left(\epsilon_{x}+1\right) \Delta x}\left(\left(\frac{\delta u}{\delta x}\right)_{i+\frac{1}{2}, j}-\left(\frac{u_{i, j}-u_{\mathrm{b}}}{\epsilon_{x} \Delta x}\right)\right)} \\
+\frac{1}{\Delta y}\left(\left(\frac{\delta u}{\delta y}\right)_{i, j+\frac{1}{2}}-\left(\frac{\delta u}{\delta y}\right)_{i, j-\frac{1}{2}}\right) .
\end{array}
$$

- Pressure gradient term:

$$
\left[\frac{\partial p}{\partial x}\right]_{i, j}=\left[\phi_{4}\left(\frac{\delta p}{\delta x}\right)\right]_{i, j}
$$

where

$$
\left[\phi_{4}\left(\frac{\delta p}{\delta x}\right)\right]_{i, j}=\frac{1}{\epsilon_{x}+0.5}\left(\epsilon_{x}\left(\frac{\delta p}{\delta x}\right)_{i+\frac{1}{2}, j}+0.5\left(\frac{\partial p}{\partial x}\right)_{\mathrm{b}}\right) .
$$

- Pressure Poisson equation:

$$
\begin{aligned}
& \frac{\Delta t}{\Delta x}\left(\left(\frac{\delta p^{n+1}}{\delta x}\right)_{i+\frac{1}{2}, j}-\left[\phi_{5}\left(\frac{\delta p^{n+1}}{\delta x}\right)\right]_{i-\frac{1}{2}, j}\right)+\frac{\Delta t}{\Delta y}\left(\left(\frac{\delta p^{n+1}}{\delta y}\right)_{i, j+\frac{1}{2}}-\left(\frac{\delta p^{n+1}}{\delta y}\right)_{i, j-\frac{1}{2}}\right) \\
& =\frac{1}{\Delta x}\left(U_{i+\frac{1}{2}, j}^{* *}-\left[\phi_{2}\left(U^{* *}\right)\right]_{i-\frac{1}{2}, j}\right)+\frac{1}{\Delta y}\left(V_{i, j+\frac{1}{2}}^{* *}-V_{i, j-\frac{1}{2}}^{* *}\right),
\end{aligned}
$$

where

$$
\left[\phi_{5}\left(\frac{\delta p}{\delta x}\right)\right]_{i-\frac{1}{2}, j}=\frac{\epsilon_{x}-0.5}{\epsilon_{x}+0.5}\left(\frac{\delta p}{\delta x}\right)_{i+\frac{1}{2}, j} .
$$


- Velocity correction:

$$
\begin{aligned}
u_{i, j}^{n+1} & =u_{i, j}^{* *}-\Delta t\left[\phi_{4}\left(\frac{\delta p^{n+1}}{\delta x}\right)\right]_{i, j}, \\
U_{i-\frac{1}{2}, j}^{n+1} & =U_{i-\frac{1}{2}, j}^{* *}-\Delta t\left[\phi_{5}\left(\frac{\delta p^{n+1}}{\delta x}\right)\right]_{i-\frac{1}{2}, j} .
\end{aligned}
$$

Eqs. (15) (18) are substituted into Eq. (7), and the velocities in the boundary cell (i.e., the $(i, j)$ cell in Fig. 1) are obtained directly, rather than by the interpolation of Eq. (13). In this sense, the momentum equation is solved in the immediate vicinity of the fluid-solid interface, while the cell-face variables are obtained by interpolation/extrapolation. The discretisations of the pressure equation (Eq. (20)) and velocity correction (Eqs. (22) and (23)) are the same as Ikeno and Kajishima [7], which guarantees that the velocity and pressure fields are consistent for this Case 2 by considering the distance to the interface from the cell centre. For this reason, the coefficient matrix for the pressure Poisson equation (see left-hand side of Eq. (20)) is no longer symmetric.

When a boundary cell of nearly-zero distance to the interface $\left(\epsilon_{x} \simeq 0\right)$ exists, $\epsilon_{x}$ in the denominators in Eqs. (15) and (17) yields large values for the convective and viscous terms. To avoid the singularity in this situation, Case 2 sets up a criterion [8]: given a threshold of $\epsilon_{\text {lim }}$ as a nearly-touching interface (or, sometimes "centre-skimming" interface when it moves) in the boundary cell where $\epsilon_{x} \leq \epsilon_{\lim }$ is satisfied, the N-S discretisation is switched to the linear interpolation, Eqs. (13) and (14), to stably obtain a predicted velocity field. For the treatments of the pressure equation and the velocity correction in the boundary cells of $\epsilon_{x} \leq \epsilon_{\mathrm{lim}}$, the discretisation schemes at the boundary cells, Eqs. (20) and (22), remain the same, since those schemes are not singular with respect to $\epsilon_{x}$. The value of $\epsilon_{\lim }$ is given in the following section.

\subsubsection{Case 3: Case 2 with extended stencils for moving boundary problem}

In a boundary cell of $\epsilon_{x} \leq \epsilon_{\text {lim }}$, the above Case 2 adopts the linear interpolation schemes (Eqs. (13) and (14) for determining the velocities in the $(i, j)$ cell), rather than solving the N-S equation in the centreskimming boundary cell. In this Case 3, a different strategy with extended stencil is employed for avoiding the singularity as well as for maintaining the consistency. The discretisations for the convective and viscous terms are modified as follows in the cells under the above distance criterion:

- Convective term

$$
\begin{aligned}
{\left[\frac{\partial(U u)}{\partial x}+\frac{\partial(V u)}{\partial y}\right]_{i, j}=} & \frac{1}{\Delta x}\left(U_{i+\frac{1}{2}, j} \frac{u_{i, j}+u_{i+1, j}}{2}-U_{i-\frac{1}{2}, j}\left[\phi_{6}(u)\right]_{i-\frac{1}{2}, j}\right) \\
& +\frac{1}{\Delta y}\left(V_{i, j+\frac{1}{2}} \frac{u_{i, j}+u_{i, j+1}}{2}-V_{i, j-\frac{1}{2}} \frac{u_{i, j}+u_{i, j-1}}{2}\right)
\end{aligned}
$$

where

$$
\left[\phi_{6}(u)\right]_{i-\frac{1}{2}, j}=\frac{\left(\epsilon_{x}-0.5\right) u_{i+1, j}+1.5 u_{\mathrm{b}}}{\epsilon_{x}+1}
$$


- Viscous term

$$
\begin{array}{r}
{\left[\frac{\partial^{2} u}{\partial x^{2}}+\frac{\partial^{2} u}{\partial y^{2}}\right]_{i, j}=\frac{1}{\left(\epsilon_{x}+0.5\right) \Delta x}\left(\left(\frac{\delta u}{\delta x}\right)_{i+\frac{1}{2}, j}-\left(\frac{\partial u}{\partial x}\right)_{\mathrm{b}}\right)} \\
+\frac{1}{\Delta y}\left(\left(\frac{\delta u}{\delta y}\right)_{i, j+\frac{1}{2}}-\left(\frac{\delta u}{\delta y}\right)_{i, j-\frac{1}{2}}\right)
\end{array}
$$

where

$$
\left(\frac{\partial u}{\partial x}\right)_{\mathrm{b}}=\frac{\left(\epsilon_{x}+2\right)^{2} u_{i+1, j}-\left(\epsilon_{x}+1\right)^{2} u_{i+2, j}-\left(2 \epsilon_{x}+3\right) u_{\mathrm{b}}}{\left(\epsilon_{x}+1\right)\left(\epsilon_{x}+2\right) \Delta x}
$$

The other discretisation schemes are the same as Case 2. Note that the treatment of Eq.(27) do not destroy the diagonally-dominant condition for discretising Eq.(26) for the Crank-Nicolson method.

This Case 3 is a thorough implementation of the direct discretisation even under a centre-skimming situation in the vicinity of the object surface by employing a wider stencil. In case of a boundary segment intersecting with both the $x$ - and $y$-grid lines and either (or both) of $\left(\epsilon_{x}, \epsilon_{y}\right)$ is smaller than $\epsilon_{\text {lim }}$, the stencil is extended in the directions away from the centre and the intersecting points (e.g., the $+x$ and $+y$ directions for Fig. 2).

\subsubsection{Pressure boundary condition}

Here, let us discuss the pressure boundary condition in the consistent direct discretisation. The following note on the independence of the pressure boundary condition in the velocity-pressure consistency applies to both Cases 2 and 3.

Because a collocated grid is employed in the present study, an interpolation is necessary to specify the pressure gradient on the cell centre. Eq.(19) includes the pressure gradient on the object boundary, while Eq.(21) does not specify $(\partial p / \partial x)_{\mathrm{b}}$. In the practical implementation, the boundary pressure gradient $(\partial p / \partial x)_{\mathrm{b}}$ in Eq.(19) is set to be 0 . This is a simplified treatment and there may be more accurate boundary conditions (e.g., [13]). However, this difference in the treatment of the boundary pressure gradient does not violate the consistency between the velocity and pressure fields as explained in the following.

Here let us consider a situation where the cell-face velocities and the pressure gradient at the cell face of the boundary cell $(i-1 / 2, j)$ in Fig 1 are given as follows (instead of Eqs.(14) and (21)):

$$
\begin{aligned}
U_{i-\frac{1}{2}, j}^{* *}=\left[\phi_{2}^{\prime}\left(U^{* *}\right)\right]_{i-\frac{1}{2}, j} & =\frac{1}{\epsilon_{x}+0.5}\left(\left(\epsilon_{x}-0.5\right) U_{i+\frac{1}{2}, j}^{* *}+u_{\mathrm{b}}^{* *}\right) . \\
{\left[\phi_{5}^{\prime}\left(\frac{\delta p}{\delta x}\right)\right]_{i-\frac{1}{2}, j} } & =\frac{1}{\epsilon_{x}+0.5}\left(\left(\epsilon_{x}-0.5\right)\left(\frac{\delta p}{\delta x}\right)_{i+\frac{1}{2}, j}+\left(\frac{\partial p}{\partial x}\right)_{\mathrm{b}}\right) .
\end{aligned}
$$


where $u_{\mathrm{b}}^{* *}$ is the intermediate fluid velocity on the object surface. By substituting the above equations into the pressure Poisson equation (20), replacing $\phi_{2}$ and $\phi_{5}$ with $\phi_{2}^{\prime}$ and $\phi_{5}^{\prime}$, respectively, the following pressure equation is obtained:

$$
\begin{aligned}
& \frac{\Delta t}{\Delta x}\left(\left(\frac{\delta p}{\delta x}\right)_{i+\frac{1}{2}, j}-\frac{\epsilon_{x}-0.5}{\epsilon_{x}+0.5}\left(\frac{\delta p}{\delta x}\right)_{i+\frac{1}{2}, j}\right)+\frac{\Delta t}{\Delta y}\left(\left(\frac{\delta p}{\delta y}\right)_{i, j+\frac{1}{2}}-\left(\frac{\delta p}{\delta y}\right)_{i, j-\frac{1}{2}}\right) \\
& =\frac{1}{\Delta x}\left(U_{i+\frac{1}{2}, j}^{* *}-\frac{1}{\epsilon_{x}+0.5}\left(\left(\epsilon_{x}-0.5\right) U_{i+\frac{1}{2}, j}^{* *}+u_{\mathrm{b}}^{* *}-\Delta t\left(\frac{\partial p}{\partial x}\right)_{\mathrm{b}}\right)\right)+\frac{1}{\Delta y}\left(V_{i, j+\frac{1}{2}}^{* *}-V_{i, j-\frac{1}{2}}^{* *}\right) .
\end{aligned}
$$

We can reasonably assume the boundary velocity of the fluid $u_{\mathrm{b}}^{n+1}$ to be time-updated from its intermediate boundary velocity $u_{\mathrm{b}}^{* *}$ as

$$
u_{\mathrm{b}}^{n+1}=u_{\mathrm{b}}^{* *}-\Delta t\left(\frac{\partial p}{\partial x}\right)_{\mathrm{b}} .
$$

Then, the above pressure equation coincides with Eq.(20). This result suggests that, in the situation of Eq. $\left(21^{\prime}\right)$, the mass conservation should be coupled with Eq. $\left(14^{\prime}\right)$ in terms of the consistency between the incompressible velocity and pressure fields.

In the present methods (Eqs.(15) $\sim(27)$ ), however, $u_{\mathrm{b}}$ is already time-updated as the membrane velocity to the $(n+1)$-level. Therefore, the above discussion means that, with the updated boundary velocity $u_{\mathrm{b}}^{n+1}$, the associated interpolated cell-face velocity by $\phi_{2}$ (i.e., Eq.(14) for Eq.(20)) and the interpolated boundary pressure gradient by $\phi_{5}$ (i.e., Eq.(21)) constitute a consistent system. In the above discussion, the pressure boundary condition at the membrane surface in Eq.(19) is formally independent of the consistency between the velocity and pressure fields.

\subsubsection{Treatment of jump cell (common to Cases 1 to 3)}

Another issue related to a moving boundary is the forcing treatment when the interface travels jumping over the centre of the boundary cell within one time step. As schematically shown in Fig. 3, the centre of the $(i, j)$ cell initially belonging to the stencil of the front side (i.e., the right-hand side) of the interface immediately changes to that in the rear side (i.e., the left-hand side). Hereafter, the cell that switches the stencils as the interface travels is referred to as "jump cell".

In the beginning of $(n+1)$-st time step of the fluid phase, the velocity and pressure at the jump cell $(i, j)$ do not reflect the already-updated position of the interface, $\boldsymbol{X}^{n+1}$, but still assigned with the values of the fluid in the front side of the membrane (see Fig. 3). With no consideration of the stencil switch (i.e., keeping the values in the right-hand side of the cell $(i, j)$ ), the values at the jump cell will be inconsistent with the values in the left-hand side, causing non-physical flow field. This problem will appear in all the cases of 1 to 3 .

In the present study, upon appearance of the jump cell $(i, j)$, the values of the velocity components and pressure of the jump cell are discarded before proceeding into the fluid routine at the $(n+1)$-st step. Then, 
those values in the rear side of the membrane are substituted with the interpolated values from the adjacent cell $(i-1, j)$ with a switched stencil: the interpolation of the velocity components at $(i, j)$ is given by the linear scheme (Eq. (13)) with the updated velocity $u_{\mathrm{b}}^{n+1}$ :

$$
\begin{aligned}
u_{i, j}^{\mathrm{p}} & =\left[\phi_{1}^{\mathrm{j}}\left(u^{\mathrm{p}}\right)\right]_{i, j} \\
& =\frac{1}{\epsilon_{x}^{\mathrm{L}}+1}\left(\epsilon_{x}^{\mathrm{L}} u_{i-1, j}^{\mathrm{p}}+u_{\mathrm{b}}^{n+1}\right),
\end{aligned}
$$

where $\epsilon_{x}^{\mathrm{L}} \Delta x$ is the $x$-distance from the cell centre $(i, j)$ to the membrane (see Fig. 3 ). The value of the pressure in the rear side of the membrane is extrapolated by a second-order biased difference method:

$$
\begin{aligned}
p_{i, j} & =\left[\phi_{7}(p)\right]_{i, j} \\
& =2 p_{i-1, j}-p_{i-2, j} .
\end{aligned}
$$

For time-updating the convective term by the Adams-Bashforth method, $\left[C_{k}^{n-1}\right]_{i, j}$ (see Eq. (7)) also needs to be evaluated based on the switched stencil. There may be several methods, and in the present study, $\left[\phi_{7}\left(C_{k}^{n-1}\right)\right]_{i, j}$ is used for re-evaluating the convective flux at the previous time level.

Note that the substitution function $\phi_{7}$ is used only for referring to the values at the jump cell $(i, j)$ in the convective and pressure terms for predicting $u_{i-1, j}^{\mathrm{p}}$ from the N-S equation. Meanwhile, as already explained, the predicted velocity $\boldsymbol{u}^{\mathrm{p}}$ at the jump cell $(i, j)$ is updated/finalised by the interpolation (Eq. $\left(13^{\prime}\right)$ ) between the object velocity $\boldsymbol{u}_{b}$ and the velocity at the neighbouring cell of the same side, $(i-1, j)$, and $p_{i, j}^{n+1}$ is given by solving the pressure Poisson equation. Those procedures are performed at every iteration of Crank-Nicolson method or at the end of the prediction step by the Adams-Bashforth method.

When the membrane partitions the boundary cell in more than two directions (i.e., the centre-to-centre lines in both the $x$ and $y$ directions are segmented by the membrane), the interpolated values in the respective directions are summed with the weight factor of 1/2, by following Fadlun et al. [4] and Sato et al. [8].

The fluid-solid interface is represented by a set of Lagrangian markers, and, as is usual in moving boundary problems, the Lagrangian markers do not exist on the surface point indicated by the symbol of $x$ in Figs. $1 \sim 3$. Therefore, $u_{\mathrm{b}}$ is linearly interpolated from the nearest two Lagrangian markers onto the intersecting points of the interface and the centre-to-centre line of the adjacent cells. 


\subsection{Spatial discretisation and time update of the membrane motion}

The variables on the membrane are defined on the Lagrangian markers, except for the tension that is defined in the middle of the markers.

The fluid-to-membrane force per unit length $\boldsymbol{F}$ is obtained from the difference between the fluid stresses $\boldsymbol{\sigma}$ (Eq. (3)) on both sides of the membrane:

$$
\boldsymbol{F}=\boldsymbol{\sigma}_{+} \cdot \boldsymbol{n}_{+}+\boldsymbol{\sigma}_{-} \cdot \boldsymbol{n}_{-},
$$

where $\boldsymbol{n}$ is the unit normal vector, and the suffixes " \pm " distinguish the sides of the membrane, as illustrated in Fig. 4. The fluid stress defined on the Eulerian frame is projected onto the Lagrangian markers: suppose $\boldsymbol{X}_{m}$ is the closest Lagrangian marker from the cell centre of the working cell $(i, j)$, and the components of Eq. (3) on $\boldsymbol{X}_{m}$ is evaluated by the Taylor expansion:

$$
g\left(\boldsymbol{X}_{m}\right)=g_{i, j}+\Delta X\left(\frac{\partial g}{\partial x}\right)_{i, j}+\Delta Y\left(\frac{\partial g}{\partial y}\right)_{i, j}+O\left(\Delta X^{2}, \Delta X \Delta Y, \Delta Y^{2}\right),
$$

where $g$ is either the pressure or velocity gradient, and $\Delta X$ and $\Delta Y$ are, respectively, the distances from $\boldsymbol{X}_{m}$ to $(i, j)$ in the $x$ and $y$ directions. These processes are applied for both sides of the membrane and repeated for every Lagrangian marker, and finally, the force acting on a Lagrangian marker is given by Eq. (29). For the evaluation of the derivatives in Eq.(30), the discretisation of $(\partial g / \partial y)_{i, J}(J=j-1, j$ for both sides of the membrane) is explained under the configuration of Fig. 4 where the membrane partitions the $(i, j)-(i, j-1)$ segment. In the following, $w$ is eigher $u$ or $v$.

$$
\begin{aligned}
\left(\frac{\delta}{\delta y} \frac{\delta w}{\delta x}\right)_{i, j} & \simeq \frac{1}{\Delta y}\left(-\frac{-w_{i-1, j}+w_{i+1, j}}{2 \Delta x}+\frac{-w_{i-1, j+1}+w_{i+1, j+1}}{2 \Delta x}\right)-\frac{1}{2}\left(\frac{\partial^{3} w}{\partial x \partial y^{2}}\right)_{i, j} \Delta y \\
\left(\frac{\delta}{\delta y} \frac{\delta w}{\delta y}\right)_{i, j} & \simeq \frac{w_{i, j}-2 w_{i, j+1}+w_{i, j+2}}{\Delta y^{2}}-\left(\frac{\partial^{3} w}{\partial y^{3}}\right)_{i, j} \Delta y \\
\left(\frac{\delta p}{\delta y}\right)_{i, j} & \simeq \frac{-p_{i, j}+p_{i, j+1}}{\Delta x}-\frac{1}{2}\left(\frac{\partial^{2} p}{\partial y^{2}}\right)_{i, j} \Delta y \\
\left(\frac{\delta}{\delta y} \frac{\delta w}{\delta x}\right)_{i, j-1} & \simeq \frac{1}{\Delta y}\left(-\frac{-w_{i-1, j-2}+w_{i+1, j-2}}{2 \Delta x}+\frac{-w_{i-1, j-1}+w_{i+1, j-1}}{2 \Delta x}\right)+\frac{1}{2}\left(\frac{\partial^{3} w}{\partial x \partial y^{2}}\right)_{i, j-1} \Delta y \\
\left(\frac{\delta}{\delta y} \frac{\delta w}{\delta y}\right)_{i, j-1} & \simeq \frac{w_{i, j-1}-2 w_{i, j-2}+w_{i, j-3}}{\Delta y^{2}}+\left(\frac{\partial^{3} w}{\partial y^{3}}\right)_{i, j-1} \Delta y \\
\left(\frac{\delta p}{\delta y}\right)_{i, j-1} & \simeq \frac{-p_{i, j-2}+p_{i, j-1}}{\Delta x}+\frac{1}{2}\left(\frac{\partial^{2} p}{\partial y^{2}}\right)_{i, j-1} \Delta y .
\end{aligned}
$$


The numerical accuracies could be improved by employing higher-order schemes. Examples of secondorder finite-difference schemes for Eqs.(31) and (32) are constructed as follows:

$$
\begin{aligned}
\left(\frac{\delta}{\delta y} \frac{\delta w}{\delta x}\right)_{i, j} \simeq & \frac{1}{2 \Delta y}\left(-3 \frac{-w_{i-1, j}+w_{i+1, j}}{2 \Delta x}+4 \frac{-w_{i-1, j+1}+w_{i+1, j+1}}{2 \Delta x}-\frac{-w_{i-1, j+2}+w_{i+1, j+2}}{2 \Delta x}\right) \\
& -\frac{1}{6}\left(\frac{\partial^{4} w}{\partial x^{3} \partial y}\right)_{i, j} \Delta x^{2}+\frac{1}{3}\left(\frac{\partial^{4} w}{\partial x \partial y^{3}}\right)_{i, j} \Delta y^{2} \\
\left(\frac{\delta}{\delta y} \frac{\delta w}{\delta y}\right)_{i, j} \simeq & \frac{1}{\Delta y^{2}}\left(\frac{12 w_{b}}{\left(1+\epsilon_{y}\right)\left(2+\epsilon_{y}\right)\left(3+\epsilon_{y}\right)}+\frac{-5+\epsilon_{y}}{1+\epsilon_{y}} w_{j+1}+\frac{8-2 \epsilon_{y}}{2+\epsilon_{y}} w_{j+2}+\frac{-3+\epsilon_{y}}{3+\epsilon_{y}} w_{j+3}\right) \\
& +\frac{11-6 \epsilon_{y}}{12}\left(\frac{\partial^{4} w}{\partial y^{4}}\right)_{i, j} \Delta y^{2} .
\end{aligned}
$$

There may be different ways of discretisation for specifying the fluid forces on the object surface. LeVeque and $\mathrm{Li}$ [14] and $\mathrm{Li}$ [15] developed their immersed interface method by incorporating the jump values of the pressure and velocity gradient into the finite-difference formulation. Miyauchi et al. [16] proposed a different method that explicitly incorporates a jump value at the interface into the finite-element formulation on a fixed mesh. In their approach, the jump value is treated as an independent variable and solved in an implicit manner. Those ideas are even applicable to the entire discretisation procedure of the N-S terms. Incorporation of the jump values of the pressure and velocity gradients into the present finite-difference formulation in a consistent manner is the subject of ongoing study by the present authors.

For non-deformable membrane, unless the motion of the object is prescribed, the fluid force $\boldsymbol{F}$ is surface-integrated and the Newton's equation of motion for the gravity centre is solved: for the translating and angular velocities the 2nd-order Adams-Bashforth method is employed, and the position and orientation are time-updated by the Crank-Nicolson method.

For flexible membrane, on the other hand, a second-order central finite difference scheme is employed for the spatial discretisation of Eq.(4). The tension $T$ is obtained by solving the following elliptic equation [17]:

$$
\frac{\partial \boldsymbol{X}}{\partial s} \cdot \frac{\partial^{2}}{\partial s^{2}}\left(T \frac{\partial \boldsymbol{X}}{\partial s}\right)=r\left\{\frac{1}{2} \frac{\partial^{2}}{\partial t^{2}}\left(\frac{\partial \boldsymbol{X}}{\partial s} \cdot \frac{\partial \boldsymbol{X}}{\partial s}\right)-\frac{\partial^{2} \boldsymbol{X}}{\partial t \partial s} \cdot \frac{\partial^{2} \boldsymbol{X}}{\partial t \partial s}\right\}-\frac{\partial \boldsymbol{X}}{\partial s} \cdot \frac{\partial}{\partial s}(\boldsymbol{B}+\boldsymbol{F}),
$$

together with the inextensible condition, Eq. (6). The time-advancement of the positions and velocities of the Lagrangian markers on the membrane are briefly summarised by following Huang et al. [17].

1. Eq.(33) is discretised with respect to the arc length $s$ around the Lagrange marker $m$, and the tension at the $(n+1)$-st step, $T^{n+1}$, is obtained by solving the following equation:

$$
\begin{aligned}
\left(\frac{\delta \boldsymbol{X}_{m}^{n}}{\delta s}\right) \cdot \frac{\delta}{\delta s} \frac{\delta}{\delta s}\left(\left.T_{m+\frac{1}{2}}^{n+1} \frac{\delta \boldsymbol{X}^{n}}{\delta s}\right|_{m+\frac{1}{2}}\right) & \\
= & r\left\{\frac{1}{2} \frac{1}{\Delta t^{2}}\left[1-2 \frac{\delta \boldsymbol{X}_{m}^{n}}{\delta s} \cdot \frac{\delta \boldsymbol{X}_{m}^{n}}{\delta s}+\frac{\delta \boldsymbol{X}_{m}^{n-1}}{\delta s} \cdot \frac{\delta \boldsymbol{X}_{m}^{n-1}}{\delta s}\right]-\frac{\delta \boldsymbol{W}_{m}^{n}}{\delta s} \cdot \frac{\delta \boldsymbol{W}_{m}^{n}}{\delta s}\right\} \\
& -\frac{\delta \boldsymbol{X}_{m}^{n}}{\delta s} \cdot \frac{\delta}{\delta s}\left(\boldsymbol{B}_{m}^{n}+\boldsymbol{F}_{m}^{n}\right)
\end{aligned}
$$


where $\delta / \delta s$ is an operator of a second-order finite difference along the arc length, and $\boldsymbol{W}_{m}^{n}$ is the velocity of the Lagrangian marker $m$ at the time level $n$, given as follows:

$$
\boldsymbol{W}_{m}^{n}=\frac{\boldsymbol{X}_{m}^{n}-\boldsymbol{X}_{m}^{n-1}}{\Delta t}
$$

The term $(\delta \boldsymbol{X} / \delta s \cdot \delta \boldsymbol{X} / \delta s)^{n+1}$ appears as one of the discretised terms of $\partial^{2} / \partial t^{2}(\partial \boldsymbol{X} / \partial s \cdot \partial \boldsymbol{X} / \partial s)$, and it is replaced with the inextensible condition, Eq. (6), following Huang et al. [17].

2. The locations of the Lagrangian markers are updated to $\boldsymbol{X}_{m}^{n+1}$ by solving Eq. (4) discretised as:

$$
r \frac{\boldsymbol{X}_{m}^{n+1}-2 \boldsymbol{X}_{m}^{n}+\boldsymbol{X}_{m}^{n-1}}{\Delta t^{2}}=\frac{\delta}{\delta s}\left(\left.T_{m+\frac{1}{2}} \frac{\delta \boldsymbol{X}}{\delta s}\right|_{m+\frac{1}{2}}\right)^{n+1}+\boldsymbol{B}_{m}^{n}+\boldsymbol{F}_{m}^{n} .
$$

In the present study with a flexible membrane, one end of the membrane is free and the other end is fixed in space with the simply-supported (pinned) condition. The boundary conditions are given as follows:

$$
\begin{aligned}
\text { Free end } & : \frac{\partial^{2} \boldsymbol{X}}{\partial s^{2}}=\frac{\partial^{3} \boldsymbol{X}}{\partial s^{3}}=0 \\
\text { Fixed end (pinned) } & : \quad \boldsymbol{X}=\boldsymbol{X}_{0}, \quad \frac{\partial^{2} \boldsymbol{X}}{\partial s^{2}}=0
\end{aligned}
$$

where $\boldsymbol{X}_{0}$ is the position vector of the fixed end.

The solution procedures are summarised as follows. The fluid force (based on the flow field at the $n$-th step) $\boldsymbol{F}_{m}^{n}$ projected onto the Lagrangian marker $m$ by Eq. (29) is substituted into Eq. (34) to solve the tension $T$, and the positions of the Lagrangian markers are updated by Eq. (35). Then, based on the updated marker positions, the velocity and pressure fields of the fluid are solved by either of the three cases in Section 3.2.

\section{Validation}

The methods are tested by comparing with analytical and reference numerical solutions of the following four different two-dimensional flows: (1) a flat plate oscillating within its plane, (2) a corrugated plate in a channel moving at a constant speed and (3) a cylinder oscillating in a stationary fluid and (4) particle migration suspended in a shear flow. These problems commonly involve a flow induced by a moving boundary, which are selected for validating the treatments of the consistent direct discretisation near the moving boundary. The third and fourth problems do not always take exact membrane structure, but by applying the present discretisation strategies to one side of the ringed surface (except for a capsule structure enclosing a fluid), the hydrodynamic force on the object surface is evaluated and compared with the reference numerical data. For those problems, the comparisons are carried out for the above three strategies of the discretisations. 
In the following sections, the grid spacing is uniform in both the $x$ and $y$ directions, and represented by $\Delta$. Sato et al. [8] mentioned that an appropriate value for $\epsilon_{\lim }$ should be given depending on the problems, and in the present study, the limiting value of $\epsilon_{x}$ and $\epsilon_{y}$ in Cases 2 and 3 are determined to be $\epsilon_{\lim }=0.1$ from a preliminary study by changing $\epsilon_{\lim }$ in the range between 0.01 and 0.2 . Hereafter, as there is no possibility of misunderstandings, $\epsilon_{x}$ and $\epsilon_{y}$ are represented simply by $\epsilon$.

\subsection{Flow induced by an oscillating flat plate}

A flat plate is placed in a stationary fluid and externally oscillated at a constant frequency in the direction parallel to the plate. The flow field of developing boundary layer above the moving wall is determined by the balance between the external driving force and viscous diffusion (with no effect of the pressure term), and the analytical solution exists for this problem. Although the numerical simulation considers the convective term, no velocity component should emerge in the wall-normal direction, which eventually produces very little numerical error in the velocity in that direction. In addition, no jump cell takes place in this problem. Therefore, the problem is suited for validation of the jump-free treatments in the vicinity of the moving wall that partitions the boundary cells to be $\epsilon \leq \epsilon_{\mathrm{lim}}$.

As shown in the schematic of the computational domain in Fig. 5, the $x$ axis is taken along the flat plate. The plate is approximately centred in the $y$ direction of the computational domain, but slightly displaced by $\eta \Delta(<\Delta / 2)$ along the $y$-axis in the middle of the boundary cell, so that the plate surface does not align with the fluid cell face and centre. Two patterns of the $\eta$-displacement are tested: Configuration 1 as $\eta=0.499$ and Configuration 2 as $\eta=0.4$, and the corresponding distances between the plate and the cell centre are $\epsilon=0.01$ and 0.1 , respectively. As both configurations satisfy $\epsilon \leq \epsilon_{\text {lim }}$, Cases 2 and Case 3 adopt different algorithms for the interpolation in the boundary cells. Note that, in Case 2, the one side of the plate employs the direct discretisation, while the discretisation in the other side (i.e., the side including the centre of the boundary cell) turns out to be the same as that of Ikeno and Kajishima [7].

The velocity of the plate is prescribed by the following equation:

$$
u=U_{0} \cos \omega t
$$

where $U_{0}$ is the velocity amplitude and $\omega$ is the angular velocity. Initially, the fluid is stationary, and the motion of the plate is given impulsively. The periodic and traction-free boundary conditions are imposed in the $x$ and $y$ directions, respectively. The Reynolds number, based on $U_{0}$ as the reference velocity and the reference length $L$ to be the travel distance of the plate in one period $\left(4 U_{0} / \omega\right)$, is set to be 100 , and $\omega$ is determined accordingly. The time increment is $\Delta t /\left(L / U_{0}\right)=1 \times 10^{-3}$, and the spatial resolutions are varied in the following range: $\Delta / L=0.1,0.05,0.033,0.025,0.01$ and 0.005 .

Assuming that the flow is homogeneous in the $x$ direction and $u \rightarrow 0$ as $|y| \rightarrow \infty$, the following analytical solution of the velocity field is obtained [18]:

$$
u_{\mathrm{th}}=U_{0} e^{-k\left|y-y_{0}\right|} \cos \left(\omega t-k\left|y-y_{0}\right|\right),
$$


where $k=\sqrt{\omega / 2 \nu}$ and $y_{0}=L-\eta \Delta$.

Figure 6 shows the grid convergence of the simulated velocity profile for Configuration 1 against the theoretical profile. The numerical error is averaged in the time range of $5 \leq t \leq 10$ after reaching quasisteady state and evaluated with the $L^{2}$ and $L^{\infty}$-norms. From Fig. 6, Case 1 exhibits about first-order convergences with respect to $\Delta$ for both $L^{2}$ and $L^{\infty}$-norms. In Case 2, the $L^{2}$ and $L^{\infty}$-norms of the errors show about 1.4-th and 1.2-nd orders of convergence, respectively, whereas the method of Case 3 takes about 1.7-th and 1.4-th orders for the $L^{2}$ and $L^{\infty}$-norms, respectively. Note that the result of Case 3 shows about the same error level as Case 1 in the lower resolution range, and gradually cuts the error to the same level as Case 2 as the spatial resolution is increased. The reason for the relatively large errors for Case 3 in the lower resolution range is that, due to the extended stencil for a cell of $\epsilon \leq \epsilon_{\lim }$, the sharp change in the developing boundary layer was not captured. Eq. (37) suggests that the velocity component attenuates exponentially in the $\pm y$ direction, and in the present problem set-up, the amplitude of the velocity component $0.25 \mathrm{~L}$ away from the oscillating plate in the normal direction decays to about $0.01 U_{0}$. Under a low spatial resolution, only a several grid points exist within the normal distance of $0.25 \mathrm{~L}$. Therefore, the treatment of extending the stencil in the boundary cell (Case 3) is not suitable to resolve the boundary layer under the low resolution cases. On the other hand, the error in Case 3 is expected to decrease with the increase in the spatial resolution, and the higher convergence rate eventually shows even a smaller error than Case 2 in $L^{\infty}$-norm for the spatial resolution smaller than $\Delta / L=1 \times 10^{-2}$.

In Configuration 1, as the distance $\epsilon \Delta$ is sufficiently small, the emerging errors due to the interpolation procedures of the velocities (for Cases 1 and 2) in the boundary cells of $\epsilon \leq \epsilon_{\lim }$ are found to be relatively small. Therefore, by taking the maximum possible gap distance $\eta=0.5-\epsilon_{\lim }$ in Configuration 2 , the effect of the interpolation strategy is examined in the following.

Figure 7 shows the convergence of the numerical errors for Configuration 2 with respect to the spatial resolution in the $L^{2}$ and $L^{\infty}$-norms. In comparison to Fig. 6, the decreasing trend of the errors in Case 3 is different from that in Configuration 1. In the lower resolution range $(\Delta / L \geq 0.05)$, the result of Case 3 is about the same error level as Case 1 , while in the higher resolution range $(\Delta / L<0.05)$ the error level is below Case 2. The larger error in the lower resolution range of Case 3 is caused by the same reason as in Configuration 1 (i.e., the poor resolution for capturing the boundary layer). On the other hand, at sufficiently fine spatial resolutions, Case 3 shows the sufficient accuracy for evaluating the viscous term in a boundary cell of $\epsilon \leq \epsilon_{\lim }$, resulting in a better accuracy than the method employing the linear interpolation (Case 2) in that cell.

The above results suggest that Cases 2 and 3 employing the direct discretisation of the viscous term in the boundary cell can attain better accuracies than Case 1 where the viscous term is calculated with the linearly-interpolated velocities. Therefore, aiming the validation of the discretisation of the viscous term in this problem, the result indicates that the direct discretisation effectively improves the numerical accuracy.

Case 3 attains a better accuracy than Case 2 under a situation when the clipping distance $\epsilon$ is around the limiting value and a sufficiently fine mesh is employed. Otherwise, the discretisation strategy of Case 2 
shows decrease in error at a constant rate and sufficient accuracy over the range of the grid spacings, suggesting that the treatment for the $\epsilon^{-1}$ terms (i.e., the threshold for $\epsilon$ and the switch of the scheme to the linear interpolation) works effectively. However, with a sufficiently fine grid spacing that captures the boundary layer, the discretisation of Case 3 gives better accuracy.

\subsection{Flow induced by a moving corrugated plate}

The second problem is a flow around a curved object travelling in a fluid at a constant speed, as schematically shown in Fig. 8. The corrugated plate of sinusoidal geometry is placed at the centre of the domain bounded by the stationary flat solid walls, and the object is towed at a constant speed $U_{0}$ in the $+x$ direction. The geometry and motion are prescribed as follows:

$$
h(x, t)=h_{0}+\delta h_{0} \cos \left(k\left(x-U_{0} t\right)\right),
$$

where $h_{0}$ is the half channel width, $k$ the wavenumber and $\delta$ the non-dimensional parameter between 0 and 1 , such that the amplitude of the sinusoidal geometry $\delta h_{0}$ varies between 0 and $h_{0}(=H / 2)$.

The periodic boundary condition is imposed in the $x$ direction, and no-slip condition is imposed on the top and bottom flat walls. The wave number $k$ is set to be $2 \pi / 5 H$ such that one wave length is accommodated in the domain width $W=5 H$. In this study, the parameter $\delta$ is set to be 0.15 , unless specified otherwise, and the amplitude of the sinusoidal geometry $\delta h_{0}=0.075 H$ is sufficiently small in comparison to the wave length. The Reynolds number, based on the average friction velocity and the half channel width is set to be 1.5. Initially, the fluid is stationary and the corrugated plate is impulsively driven at the constant speed. At the external domain boundaries, the Neumann condition is imposed for the pressure.

This problem is characterised by the flow field along a weakly-undulating geometry in the $x$ direction, where the intersecting angle between the interface and the cell face varies (unlike the problem in $\S 4.1$ ). Although the local geometric change in the $y$ direction is small in comparison to the change in the longitudinal direction, the treatment of the jump cells accounts for the numerical result in this problem.

An approximate analytical solution is constructed under the assumptions that (a) the lubrication approximation for fluid is applicable under the condition of $k h_{0} \ll 1$ and (b) a rectangle approximation is applicable for the respective regions below and above the plate (hereafter, the lower and upper regions, respectively) under the condition of $\delta \ll 1$.

In the lower region, the lubricated analytical solution of the velocity components in the $x$ and $y$ directions, $u_{\mathrm{th}}$ and $v_{\mathrm{th}}$, respectively, are constructed as follows:

$$
\begin{aligned}
& u_{\mathrm{th}}(x, y, t)=R \frac{y(h-y)}{h^{3}}+3 U_{0} \frac{y(h-y)}{h^{2}}-U_{0} \frac{h-y}{h}+U_{0} \\
& v_{\mathrm{th}}(x, y, t)=\left(R \frac{y(h-y)}{h^{3}}+2 U_{0} \frac{y(h-y)}{h^{2}}\right) \frac{y}{h} \frac{\partial h}{\partial x},
\end{aligned}
$$


where

$$
R=-\frac{3 U_{0} h_{0}\left(1-\delta^{2}\right)}{1+\delta^{2} / 2}
$$

As the flow phenomena in the upper and lower sides of the corrugated plate are the same (but with a phase difference by $\pi$ ), only the flow in the lower region is taken for the error analysis in the following. Hereafter, the phase angle is represented by $\varphi=k\left(x-U_{0} t\right)$.

Figure 9 plots the instantaneous velocity vectors obtained by Case 2. The spatial resolution and time increment are chosen as $\Delta / H=0.025$ and $\Delta t /\left(H / U_{0}\right)=5 \times 10^{-5}$, respectively. Note that the projected thickness of the corrugated plate is $2 \delta h_{0}=0.15 H$, and therefore this weakly-undulating geometry lies over six computational cells. The velocity vectors in the upper region of the corrugated plate are plotted at every 10 grid points in the $x$ direction. In the lower region of the plate, the velocity profiles are shown at four characteristic locations in the $x$ direction; the points of the most narrow and wide channel widths $(\varphi=0, \pi)$ and the intermediate axial positions $(\varphi=\pi / 2,3 \pi / 2)$. From the velocity vectors, the flow is accelerated in the regions where the channel widens in the moving direction, and decelerated where it contracts. And for all the axial locations, the velocity profiles are in good agreement with the analytical solution by the lubrication assumption, as the present consistent direct discretisations (Cases 2 and 3) have sufficiently sharp representation of the interface.

The velocity distributions in the $y$ direction in Fig. 9 take concave and convex profiles. In between the concave and convex velocity profiles, two axial locations of a nearly-linear velocity profile exist. The necessary condition for the linear velocity profile is obtained from Eq. (39a) as $R / h+3 U_{0}=0$, and the corresponding phase angles are obtained as

$$
\varphi=\arccos \left(-\frac{3 \delta}{2+\delta^{2}}\right)
$$

Interestingly, the phase angle of the linear profile approaches $\pi / 2$ as $\delta \rightarrow 0$. However, the situation of $\delta=0$ corresponds to a flat wall; therefore, the linear velocity profile actually appears everywhere. Meanwhile, $\varphi \rightarrow \pi$ as $\delta \rightarrow 1$ means that the lubrication assumption becomes doubtful for the largely undulating geometry of the plate. In the present situation, the amplitude parameter $\delta$ is 0.15 , and the linear velocity profiles should appear at $\varphi \fallingdotseq 0.57 \pi, 1.57 \pi$, which are close to the locations of the nearly-linear crosssectional velocity profiles in Fig. 9.

Figure 10 shows the grid dependence of the velocity to the analytical solution. The errors are evaluated by the $L^{2}$ and $L^{\infty}$-norms after reaching quasi-steady state. The figure shows that, for all the cases, the both $L^{2}$ and $L^{\infty}$ errors tend to decrease by decreasing the grid spacing, while the decrease rate is rather saturated at the highly resolved conditions.

This is probably because the assumptions for the approximate solution Eq.(39). To check the effective range of the lubrication assumption, $\delta$ is decreased to 0.10 . Figure 11 shows the error trends of $u$ in the range of $\Delta \leq 2 \delta h_{0}$ (i.e., the projected thickness of the corrugation). The graph shows an improved error 
level (with respect to the lubrication solution) with this reduced amplitude $\delta$. Therefore, the numerical error is readily decreased by imposing an ideal situation for the lubrication assumption.

The trend of the grid convergence of the pressure gradient is also investigated for the same geometry as Fig. 9. The approximate analytical solution of the pressure gradient due to the moving corrugated plate is given as:

$$
-\frac{\partial p}{\partial x}=\frac{6 \mu_{f} U_{0}}{h^{2}}\left(\frac{1-\delta^{2}}{1+\delta^{2} / 2} \frac{h_{0}}{h}-1\right)
$$

in the lower-half region of the plate, where $\mu_{f}$ is the viscosity. Figure 12 summarises the result. For all the cases, except at the lowest spatial resolution $(\Delta / H=0.167)$, the errors decrease with the spatial resolution. At $\Delta / H=0.167$, the numerical accuracies for all the cases are apparently improved. This is because, at this lowest spatial resolution, the grid spacing is larger than the maximum displacement of the undulation (twice the undulation amplitude, $2 \delta h_{0}=0.15 H$ ): the plate moves along a path between two cell centres (i.e., the width is $\Delta y=0.167 H$ ), and therefore, no jump cell takes place in both $x$ and $y$ directions. Due to this situation of the substantial insufficiency in resolution, the balance between the pressure gradient and the wall shear stresses simply determines the flow (with no spurious pressure oscillations, as explained later), and therefore, the computation apparently yields the reduced error in the pressure gradient.

\subsection{Flow induced by an oscillating cylinder}

In the previous subsections, the effect of the object on the shear-driven fluid flow has been studied. To assess the validity of the fluid force acting on the object, the flow induced by a cylinder oscillating in a stationary fluid is simulated, and the pressure fluctuation in association with the appearance of jump cell due to the motion of the object is discussed.

A schematic of the flow field is shown in Fig. 13. The diameter of the cylinder is set to $D$. The domain size is $50 D$ and $30 D$ in the $x$ and $y$ directions, respectively, and a stationary fluid is given as the initial condition. For the boundary conditions, the gradient-free condition is given for the velocity components in the $x$ direction, and a traction-free boundary condition in the $y$ direction is imposed with $p=0$ (Dirichlet boundary condition), assuming that the top and bottom boundaries are far enough from the oscillating cylinder. The cylinder motion is prescribed as follows:

$$
x_{c}=x_{0}-A \sin (2 \pi f t),
$$

where $x_{0}(=25 D)$ is the neutral position, $A$ the amplitude of oscillation and $f$ the frequency. The relative distances of the Lagrangian markers on the cylinder surface are set to be about the same as the grid spacings.

The flow field of this problem is characterised by two non-dimensional numbers: Reynolds number $\left(\operatorname{Re}=U_{\max } D / \nu\right)$ and Keulegan-Carpenter $\left(\mathrm{KC}\right.$, hereafter) number $\left(\mathrm{KC}=U_{\max } / f D\right)$, where $U_{\max }$ is the maximum travelling speed of the cylinder, $2 \pi f A$. 
Present simulation result is compared with that of Dütsch et al. [19] who employed a body-fitted coordinate system around the cylinder. Therefore, by adjusting the simulation condition to that of Dütsch et al. [19], the two non-dimensional numbers are set to $\mathrm{Re}=100$ and $\mathrm{KC}=5$.

Figure 14 plots the instantaneous flow fields (after reaching quasi-steady state) near the cylinder. Here, the grid spacing and time increment are $\Delta / D=0.02$ and $\Delta t /\left(D / U_{\max }\right)=2.5 \times 10^{-3}$, respectively. The velocity and pressure fields in Fig. 14(a) are shown for the moment when the cylinder is on the move at $U_{\max }$ in the $+x$ direction (i.e., when the phase angle of the oscillation is $\pi$ ). The velocity vectors show the significant change in the flow field around the cylinder, especially a vortical structure in the trailing-edge side. This observation coincides with the pressure distribution shown in the lower-half region of the figure. In Fig. 14(b), the profiles of the axial and transverse velocity components are shown at three different axial locations: a cross-section cutting through the cylinder centre, and $0.1 D$ up- and down-stream sides from the leading and trailing edges. The symbols are the numerical result by Dütsch et al. [19], and the lines are those by Case 2 of the present method. The figure shows that the results from the present simulation are in good agreement with those obtained using a body-fitted coordinate system. Our preliminary study shows that the similar velocity and pressure profiles are observed for Case 3, while large discrepancies are observed for the velocity profiles with Case 1 in those cross-sections (figure omitted). This is because, in Case 1, the velocity field corrected by the pressure field does not satisfy the no-slip condition exactly at the object surface, causing a slight imbalance with the cylinder velocity.

To check the fluid force on the object surface, the fluid stress $\sigma$ is projected onto the Lagrangian markers as explained in Eq. (30), and drag force on the moving cylinder is calculated.

The time history of the drag coefficient is compared with that of Dütsch et al. [19] in Fig. 15. Case 1 exhibits strong fluctuation of the drag coefficient with time, and the preliminary study shows that the increase in the spatial resolution $(\Delta / D=0.02$ and 0.0125$)$ does not improve this fluctuating trend. On the other hand, the results by Cases 2 and 3 show relatively small fluctuation, and along with the preliminary observations at $\Delta / D=0.02$ and 0.04 , increasing the spatial resolution successively suppress the temporal fluctuations in the drag coefficient.

Figure 16 plots the total variation of the drag coefficient from the result of Dütsch et al. [19] against the spatial resolution. The figure shows that the variation in the result of Case 1 is less sensitive to the spatial resolution, while the variation in the results by Cases 2 and 3 exhibit monotonous decreases with the resolution. This error-decreasing trend suggests that, given an appropriate time increment under sufficiently fine spatial resolution, the present discretisation strategies (Cases 2 and 3) are applicable to fluid-structure interaction problems. 


\subsection{Particle migration in a shear flow}

In the previous subsections, the motions of the object are prescribed. To apply the method to a problem of fluid-driven motion, migration of particle in a shear flow is studied. Only Case 3 is employed as a fully consistent case.

Figure 17(a) shows a schematic of the problem. A single rigid particle (i.e., a finite-volume object) of diameter $D$ is placed in wall-driven shear flow in a 2-D channel of width $L=4 D$. The bulk Reynolds number, $\operatorname{Re}_{\mathrm{b}}=U L / \nu$, is 40 . The initial position of the particle centre is at $y=D$, and the particle is released into the flow with zero translating and angular velocities, i.e., with a finite velocity gap $\delta \mathrm{V} / U=$ $0.25 \times(2-y / D)(0.5 \leq y / D \leq 1.5)$ against the background shear flow. The particle is rigid and it has the same density as the ambient fluid (neutrally-buoyant). The velocity distribution inside the rigid particle is given by $\boldsymbol{u}=\boldsymbol{v}_{p}+\boldsymbol{r} \times \boldsymbol{\omega}_{p}$, where $\boldsymbol{v}_{p}$ and $\boldsymbol{\omega}_{p}$ are the translating and angular velocities, and $\boldsymbol{r}$ is the radial vector from the particle centre $(|\boldsymbol{r}| \leq D / 2)$. The spatial resolution is $\Delta / D=0.0125$, and the spacings between the equally-arranged Lagrangian markers on the particle surface is $\Delta s / D=0.0090$.

Figure 17(b) compares the time histories of the $y$-coordinate of the particle with the available numerical results in the literature [20]. The present numerical result shows good agreement with the reference data.

For comparison, a membrane of capsule structure filled with the fluid is tested. The membrane does not deform, and the fluid enclosed by the membrane has the same density and viscosity as the fluid outside. The density ratio $r$ is unity. The other computational parameters are the same, and the numerical procedure for solving the fluid inside the capsule is the same as the treatment of the fluid motion separated by the membrane in Section 4.2. In Fig. 17(b), the fluid-filled capsule exhibits slower migration than the rigid case. The reason for the different behaviours for the rigid and capsule particles is that the fluid motion inside the capsule is immediately induced by the particle rotation. Figure 18 shows the instantaneous velocity fields inside and outside the capsule at $t=60$ and 135. In the initial developing stage $(t \lesssim 100 L / U)$, a translating component is predominant for the flow inside the capsule (Fig. 18(a)), and the boundary layer is developed inside the capsule (Fig. 18(b)) from the observation on the moving frame of reference at the particle translating velocity. In the secondary developing stage $(t>100 L / U)$ as shown in Fig. 18(c), a rigid rotation of the fluid is fully developed. The above figures suggest that the rotating motion of the capsule is also influential to the lateral migration of the capsule. Figure 19 compares the angular velocities of the rigid and capsule particles against the lateral position of the particle, $y / L$. In the initial developing stage, the capsule needs less energy to revolve as it only drags the fluid near the inner surface of the capsule, while the rigid particle has to revolve the entire body. Therefore, the capsule revolves at slightly higher angular velocity than the rigid particle, resulting in the additional Magnus lift in the $-y$ direction for the flow configuration in Fig. 17(a). The difference in strength in the lift causes the capsule a slower migration towards the centreline.

Migration motion is known to show a delicate response against the slight difference in particle density. Therefore, another comparison is the motion of non-neutrally buoyant particle in a shear flow under the 
effect of the gravity. Only the case with rigid particle is studied, and no capsule particle is employed here. The gravity effect on the fluid motion is solved by giving the external force in Eq.(2), whereas the rigid particle experiences the gravity at the gravity centre (in the equation of motion). Figure 20(a) shows a schematic of the problem. The spatial resolutions of the fluid mesh and the Lagrangian markers are the same as the above neutrally buoyant case. Bulk Reynolds number $\operatorname{Re}_{\mathrm{b}}=U L / \nu$, particle Reynolds number $U(D / 2)^{2} /(\nu L)$ and Froude number $g L / U^{2}$ are set to be 40, 0.625 and 392, respectively, where $g$ is the magnitude of the gravitational acceleration. The particles are rigid, and the density ratio of the solid to the fluid $\rho_{s} / \rho_{f}$ is tested for the following two cases: 1.002 and 0.998 . The respective initial slip velocities $\delta V / U$ (at the particle centre level) are given as $0.637 \times(L / D) \operatorname{Re}_{\mathrm{b}}^{-1}$ and $0.057 \times(L / D) \operatorname{Re}_{\mathrm{b}}^{-1}$. Initially, the particle is placed at $y=0.5 \mathrm{~L}$. Figure 20(b) shows that the results of both density cases show good agreement with the reference numerical results [20].

For a wider applicable range of the density ratio, an interaction problem between a vortical flow and a 3-D rigid particle of 10 times higher density than the ambient fluid is simulated in Appendix. The validity is assessed through comparisons with independently-conducted numerical results [21].

The above results suggest that the method is applicable to fluid-particle interaction problems.

\section{Application: Flapping filament}

The method with Case 3 is coupled with the motion of a flexible filament, and applied to an interaction problem between a filament and uniform flow in two dimensions. In this section, the convective and viscous terms in Eq. (2) are time-updated by the four-stage Runge-Kutta (RK4) method, Although no difficulty arises by the semi-implicit method for the fluid phase (i.e., the Crank-Nicholson method) to perform the following simulations, the explicit time-marching method is employed to carry out the simulations within a feasible computational time.

The numerical procedure for the motion of the filament is as explained in Section 3.3. To assess the validity, the motion of a filament under the effect of the gravity (with no ambient fluid) is simulated, and the result is compared with the numerical result by Huang et al. [17]. One end of the filament is pinned and the other end is free. The number of Lagrangian markers along the filament is 50, while Huang et al. (2007) used 100 points of markers. The time increment of our simulation is $5 \times 10^{-4}$, and the other computational parameters are the same as [17]. Figure 21 is the comparison of the time evolutions of the horizontal displacement of the filament at the free end. Although the oscillation frequency is slightly different, the amplitudes of the oscillations and the shapes of the curves agree well to each other; even the agreement of the asymmetry in the displacement curve around each peak, indicating that the snapping motion of the free end is simulated. The figures suggest that the numerical method for solving the motion of the filament of Huang et al. [17] has been implemented correctly. In the following, the proposed method is applied for interaction problem between a flexible filament and a uniform flow. 
Figure 22 shows a schematic of the computational domain. As the figure shows, the natural filament length is $L$, and the computational domain size is $20 L$ and $10 L$ in the $x$ and $y$ directions, respectively. The leading edge of the filament is pinned in space, allowing rotation around the fixed marker point. A uniform flow $U_{0} \boldsymbol{e}_{x}$ is allowed to enter at the left boundary, and the convective boundary condition is given at the outgoing boundary. A traction-free boundary condition is specified at the top and bottom boundaries. The initial orientation of the filament is inclined by $0.01[\mathrm{rad}]$ against the $x$ axis. The filament is supported still for a certain period of time in the initial developing stage until the flow around the stationary filament reaches steady state, and then the interaction between the fluid flow and filament is started. In the following, $L$ and $U_{0}$ are taken as the reference length and velocity, respectively. The Lagrangian markers on the filament are arranged at the same intervals as the fluid cell size.

The numerical result is compared with the analysis for the flapping condition by Connell and Yue [11]. They showed, by their linear stability analysis, that the stable condition (i.e., no flapping condition) for a filament in a uniform flow is given with three non-dimensional numbers of Re, $r$ and $\gamma$ as

$$
r<\frac{4 \alpha \mathrm{Re}^{-0.5}+\gamma k^{2}}{1-2 \alpha \mathrm{Re}^{-0.5} k-0.5 \gamma k^{3}},
$$

where $\alpha=0.33$ and $k$ is the wavenumber of the flapping filament. Eq. (44) shows that increasing $k$ causes the increase in the right-hand side. Therefore, by specifying the smallest wavenumber, the stable limit for the density ratio is obtained.

By fixing $\gamma$ and Re to be $1 \times 10^{-5}$ and 100, respectively, numerical simulations are carried out for different values of $r$, under the spatial resolution of $\Delta / L=0.04$ and time increment of $\Delta t /\left(L / U_{0}\right)=$ $4 \times 10^{-4}$. From Eq. (44), this condition estimates a stable limit for the density ratio to be $r=0.22$. Figure 23 shows the time-evolutions of the $y$-displacements of the trailing edge for different density ratios. As $r$ is increased, the flapping amplitudes become large. The figure shows that the filament is almost stationary at $r=0.15$ and in a small flapping state at $r=0.20$, suggesting an approximate agreement with the critical density ratio predicted by Eq. (44). Note that the derivation of Eq. (44) assumes a constant tension along the infinite filament length: practically, this assumption would estimate a slightly more stable condition for flapping according to the analysis with variable tension [22].

Figure 24 shows the sequential snapshots of the velocity and pressure fields together with the corresponding geometry of the filament. The density ratio of the filament is $r=0.5$. And the time range in Fig. 24 is set $20.0 \leq t /\left(L / U_{0}\right) \leq 22.4$, where the most violent (but regular) motion of the filament tip is observed. The colour correspond to the pressure level.

The figure shows the large deviations in the velocity vectors around the trailing edge in response to the large $y$-displacements at the filament tip. Also, from the different flow patterns in the upper and lower sides of the filament, the velocity field is resolved sharply in the transverse direction across the filament. Considering the smooth evolution of the displacements in Figs. 23 and 24, the pressure fluctuations in Fig. 15 due to the moving object cause little influence on the motion of the filament. 
Strong snapping motions of the filament are observed in Figs. 24(a) and 24(c), which are also found in Fig 23 as the sharp asymmetric peaks for $r=0.50$ at around $t=20.0$. The pressure distributions in Fig. 24 exhibit sharp discontinuity across the object surface. This highlighted pressure gap across the thin object is because the method considers the position of the filament in the discretisations of the velocity prediction and the pressure Poisson equation, suggesting the importance of the direct discretisation and the consistent coupling between the incompressible velocity and pressure fields.

\section{Conclusion}

For solving a moving boundary problem with a thin membrane in a fluid on a Cartesian system, we showed the importance of (1) the consistency between the velocity and pressure fields by taking account of the distance to the object surface from the cell centre and (2) the direct discretisation of the primary variables arranged at the centre of the fixed computational cell in the Cartesian frame. A particular problem arises when a "jump cell" takes place in association with the passage of the interface across a cell centre of boundary cell within a single time step. For this problem, the discretisation stencil was switched accordingly, and the terms in the rear side of the membrane were re-evaluated with the value at the previous time level (just before the jump takes place) and incorporated into the consistent direct discretisation schemes.

The validity of the numerical results were established through comparisons with the analytical solutions of the fluid motions for such solid-induced flow problems as an oscillating flat plate and translating corrugated plate. The numerical results showed that the fluid motions in both sides of the moving interface were solved with sufficient accuracies, regardless of the implementation of a threshold for the surface distance or a wider stencil introduced in place of the threshold. However, with sufficiently fine grid spacing for capturing the boundary layer, the method with a wider stencil is recommended as this treatment constitutes the fully consistent coupling between the pressure and incompressible velocity fields. The result suggested that the two fluids in both sides of the interface were sharply separated across the thin membrane with the aid of the jump-cell treatment for the moving boundary. The fluctuation in hydrodynamic force acting on the membrane in association with the jump cells was found to be readily reduced by increasing the spatial resolution. Migration motion of the particle in a shear flow was also studied to check the interaction between the object and the fluid. The three density cases of neutrally-buoyant, slightly-heavier and -lighter particles than the ambient fluid showed good agreement with the reference numerical results. The results suggested the potential applicability to a particle-dispersed two-phase flow problem.

The method was further applied to a flapping filament in a uniform flow. Through observation of the interaction between the fluid and flexible filament, the sharp treatment of the interface was capable of capturing the fluid motions in both sides of the filament and the violent motion of the filament.

In the end, let us mention future perspectives for applying the present method to high Reynolds number flows. There may be three points to be considered for dealing with high Reynolds number flows; higher resolution, stabilisation and turbulence modelling. For the treatment of higher resolution, the present method 
can adopt a local mesh refinement around a moving membrane as the method is developed with different grid spacings in all the directions. To damp the numerical instabilities, if necessary, upwind schemes are easily constructed by considering the position of the object surface, and our preliminary study shows that an upwind scheme effectively stabilises a high-Reynolds number flow. For further high Reynolds numbers, a turbulence model is necessary. The original version of the consistent discretisation by Ikeno and Kajishima [7] was developed for large-eddy simulation around an object of non-regular geometry, and the same idea can be applied to the present method.

The present study showed the effectiveness of the consistent coupling and direct discretisation for solving the fluid motions around a moving thin structure and rigid particle, and the proposed idea is expected to promote further development of numerical analysis of multiphase flows.

\section{Acknowledgements}

The authors thank Dr. N. Sato (Toyota Central R\& D Labs. INC., Aichi, Japan) for fruitful discussion and Dr. T. Fukada (Central Research Institute of Electric Power Industry, Yokosuka, Japan) for providing the data in Appendix. This work is partly supported by Grant-in-Aid (B) No.16H04271 and No.17H03174 of the Japan Society for the Promotion of Science (JSPS). 


\section{Appendix: Particle motion in a 3-D vortical flow}

A wider applicable range of density ratio is investigated through a simulation of a flow containing a rigid particle of 10 times higher density than the ambient fluid. For this demonstration, the present method is extended to three dimensions, and an interaction problem between the Taylor-Green vortices and a 3-D rigid spherical particle is solved. The result is compared with the independently-conducted numerical results by Fukada et al. [21]. To match the same problem in their study, the following computational set-up and conditions are employed.

The velocity components of the unit Taylor-Green vortex $\boldsymbol{U}_{\mathrm{TG}}$ are represented as follows:

$$
\boldsymbol{U}_{\mathrm{TG}} \cdot \boldsymbol{e}_{x}=U \sin \left(\frac{y}{L}\right), \quad \boldsymbol{U}_{\mathrm{TG}} \cdot \boldsymbol{e}_{y}=-U \sin \left(\frac{x}{L}\right), \quad \boldsymbol{U}_{\mathrm{TG}} \cdot \boldsymbol{e}_{z}=0,
$$

where $U$ and $L$ are the velocity and the length units, and $\boldsymbol{e}_{i}(i=x, y, z)$ is the basis vector. The flow is maintained by introducing the following external forcing terms in the right-hand side of Eq.(2):

$$
\boldsymbol{f}_{\mathrm{ext}} \cdot \boldsymbol{e}_{x}=\frac{U \nu}{L^{2}} \sin \left(\frac{y}{L}\right), \quad \boldsymbol{f}_{\mathrm{ext}} \cdot \boldsymbol{e}_{y}=-\frac{U \nu}{L^{2}} \sin \left(\frac{x}{L}\right), \quad \boldsymbol{f}_{\mathrm{ext}} \cdot \boldsymbol{e}_{z}=0 .
$$

The Reynolds number is defined as $\operatorname{Re}=U L / \nu=18$. The particle diameter $D / L=2 \pi / 16$ and the density ratio of the rigid particle to the ambient fluid is 10 . The spatial resolution and the time increment are set to $D / \Delta=24$ and $(U / L) \Delta t=3.31 \times 10^{-4}$, respectively. Initially, the particle is released from the centre-coordinates of $\left(x_{c} / \pi L, y_{c} / \pi L, z_{c} / \pi L\right)=(1 / 2,1,0)$ into the flow with the same centre velocity as the local undisturbed flow velocity $\left(\left.\boldsymbol{U}_{\mathrm{TG}}\right|_{x_{c}, y_{c}, z_{c}}\right)$ and the angular velocity 0.

The centre of the solid particle is tracked in the Lagrangian way by integrating the hydrodynamic force on the outer surface of the particle (Section 3.3). For projecting the hydrodynamic force onto the particle surface, a tri-linear interpolation is employed. When the solid surface partitions the boundary cell in the three directions (i.e., tri-directionally opened boundary cell), the interpolated values in the respective directions are summed with the weight factor of $1 / 3$.

Figure 25 compares the particle trajectory by the present method (red solid line) with the other two numerical results obtained by Fukada et al. [21]; a fully-resolved simulation by an immersed boundary method [9] and a simulation based on a volume-averaged equation considering the surface stress and flow disturbance [21]. Agreement of the particle trajectories in the vortical flow is good, and the results suggests the applicability of the present method to a wider range of the density ratio. 


\section{References}

1 Peskin, C.S., "Flow patterns around heart valves: a numerical method", Journal of Computational Physics 10, pp.252-271 (1972)

2 Goldstein, D., Handler, R. and Sirovich, L., "Modeling a no-slip flow boundary with an external force field", Journal of Computational Physics 105, pp.354-366 (1993)

3 Mohd-Yusof, J., "Development of immersed boundary methods for complex geometries", Annual Research Briefs, Center for Turbulence Research, pp.325-336 (1998)

4 Fadlun, E.A., Verzicco, R., Orlandi, P. and Mohd-Yusof, J., "Combined immersed-boundary finitedifference methods for three-dimensional complex flow simulations", Journal of Computational Physics 161, pp.35-60 (2000)

5 Kim, J., Kim, D. and Choi, H., "An immersed-boundary finite-volume method for simulation of flow in complex geometries", Journal of Computational Physics 171, pp.132-150 (2001)

6 Ikeno, T. and Kajishima, T., "Difference Formula of Poisson Equation Consistent with an Immersed Boundary Method" (in Japanese), Trans. JSME Ser.B 70 No.697, pp.2239-2245 (2004)

7 Ikeno, T. and Kajishima, T., "Finite-difference immersed boundary method consistent with wall conditions for incompressible turbulent flow simulations", Journal of Computational Physics 226, pp.14851508 (2007)

8 Sato, N., Takeuchi, S., Kajishima, T., Inagaki, M. and Horinouchi, N., "A consistent direct discretization scheme on Cartesian grids for convective and conjugate heat transfer", Journal of Computational Physics 321, pp.76-104 (2016)

9 Breugem, W.-P., "A second-order accurate immersed boundary method for fully resolved simulations of particle-laden flows", Journal of Computational Physics 231, pp.4469-4498 (2012)

10 Seo, J.H. and Mittal, R., "A sharp-interface immersed boundary method with improved mass conservation and reduced spurious pressure oscillations", Journal of Computational Physics 230, pp.7347-7363 (2011)

11 Connell, B.S.H. and Yue, D.K.P., "Flapping dynamics of a flag in a uniform flow", Journal of Fluid Mechanics 581, pp.33-67 (2007)

12 Dukowicz, J.K., and Dvinsky, A.S., "Approximate fractorization as a high order splitting for the implicit incompressible flow equations", Journal of Computational Physics 102 pp.336-347 (1992) 
13 Petersson, N.A., "Stability of pressure boundary conditions for stokes and Navier-Stokes equations", Journal of Computational Physics 172, pp.40-70 (2001)

14 LeVeque, R.J. and Li, Z. "The immersed interface method for elliptic equations with discontinuous coefficients and singular sources", SIAM Journal on Numerical Analysis 31, pp.1019-1044 (1994).

15 Li, Z., "A Note on Immersed Interface Method for Three-Dimensional Elliptic Equations" Computers \& Mathematics with Applications 31, No. 3, pp. 9-17 (1996)

16 Miyauchi, S., Takeuchi, S. and Kajishima, T., "A numerical method for interaction problems between fluid and membranes with arbitrary permeability for fluid", Journal of Computational Physics $\mathbf{3 4 5}$, pp.33-57 (2017)

17 Huang, W.X., Shin, S.J. and Sung, H.J., "Simulation of flexible filaments in a uniform flow by the immersed boundary method", Journal of Computational Physics 226 pp.2206-2228 (2007)

18 White, F.M., Viscous Fluid Flow, McGraw-Hill (1974)

19 Dütsch , H., Durst, F., Becker, S. and Lienhart, H., "Low-Reynolds-number flow around an oscillating circular cylinder at low Keulegan-Carpenter numbers", Journal of Fluid Mechanics 360, pp.249-271 (1998)

20 Feng, J., Hu, H.H. and Joseph, D.D. (1994) 'Direct simulation of initial value problems for the motion of solid bodies in a Newtonian Fluid. Part 2. Couette and Poiseuille flows", Journal of Fluid Mechanics 277 pp.271-301

21 Fukada, T., Fornari, W., Brandt, L., Takeuchi, S. and Kajishima, T., ”A numerical approach for particlevortex interactions based on volume-averaged equations", International Journal of Multiphase Flow Vol. 104, pp.188-205 (2018)

http://dx.doi.org/10.1016/j.ijmultiphaseflow.2018.02.019

22 Moretti, P.M., "Tension in fluttering flags", International Journal of Acoustics and Vibration 8 No. 4 , pp.227-230 (2003) 


\section{Figures}

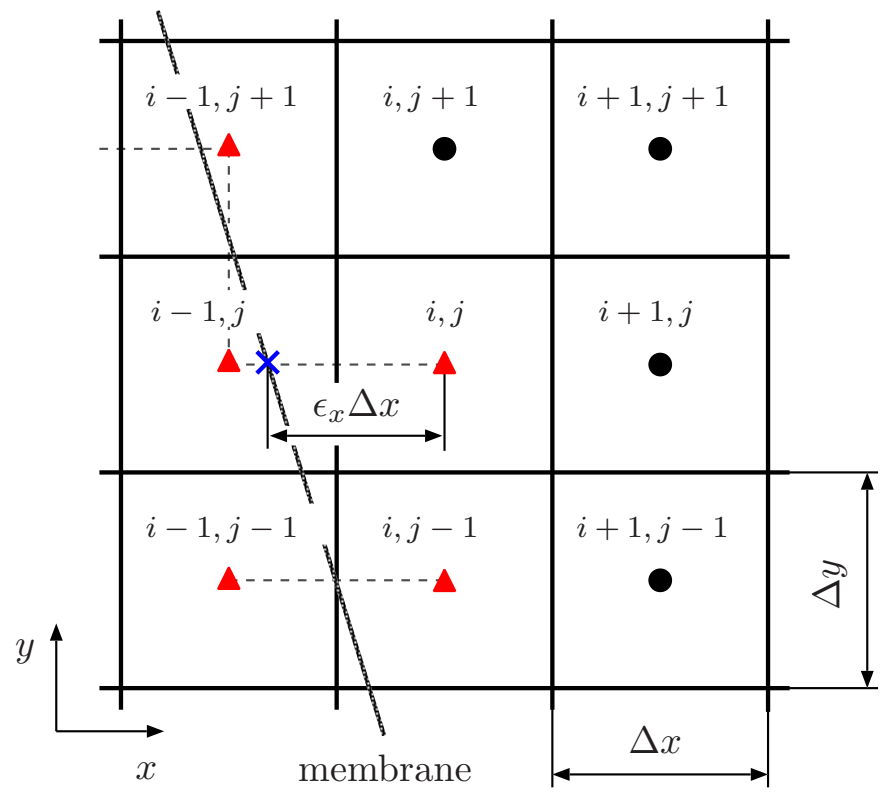

Figure 1: Configuration of a Cartesian grid and a membrane. The filled circle and filled triangle represent the fluid and boundary cells, respectively, and the cross symbol shows the immersed point.

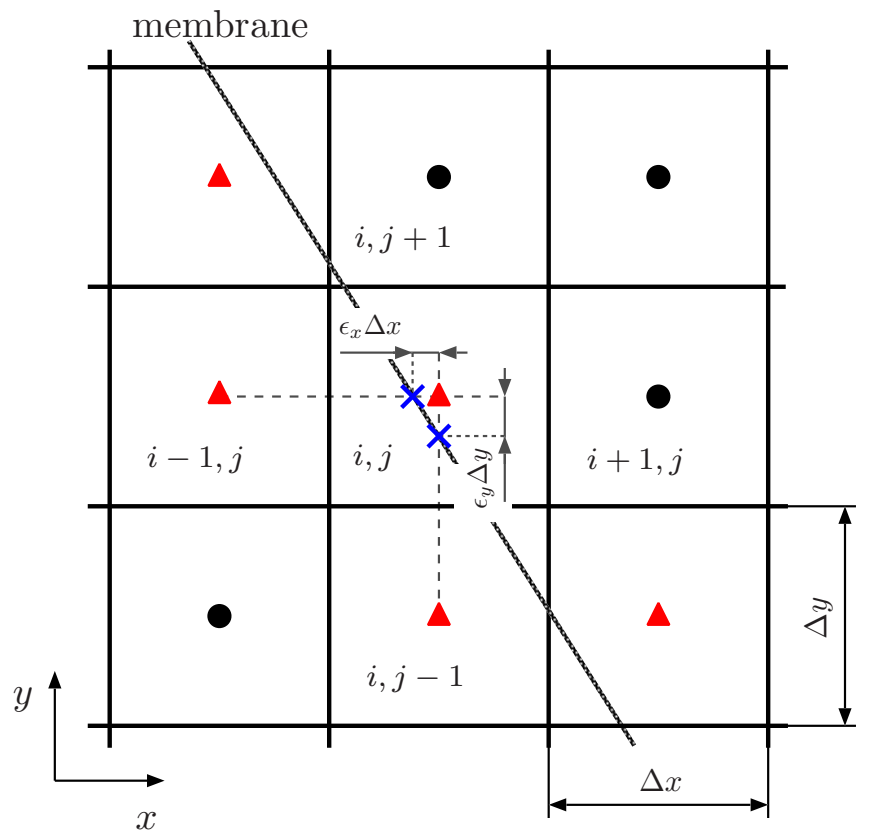

Figure 2: A situation of a cell-centre nearly touching the membrane surface. 

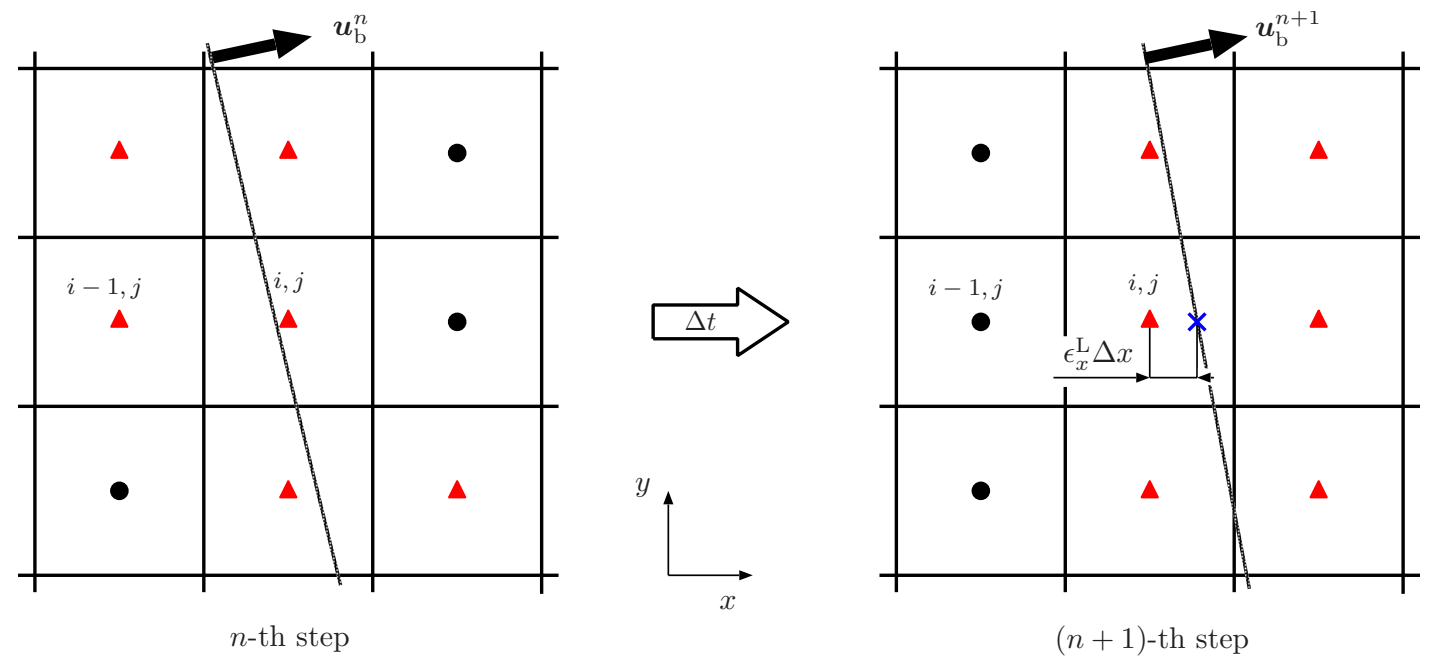

Figure 3: Change in the positional context between the cell centre $(i, j)$ and the interface when the membrane crosses the cell centre within one time step. Upon appearance of the jump cell $(i, j)$ in the left-hand side of the membrane, the switch of the stencil needs to be updated for the discretisation.

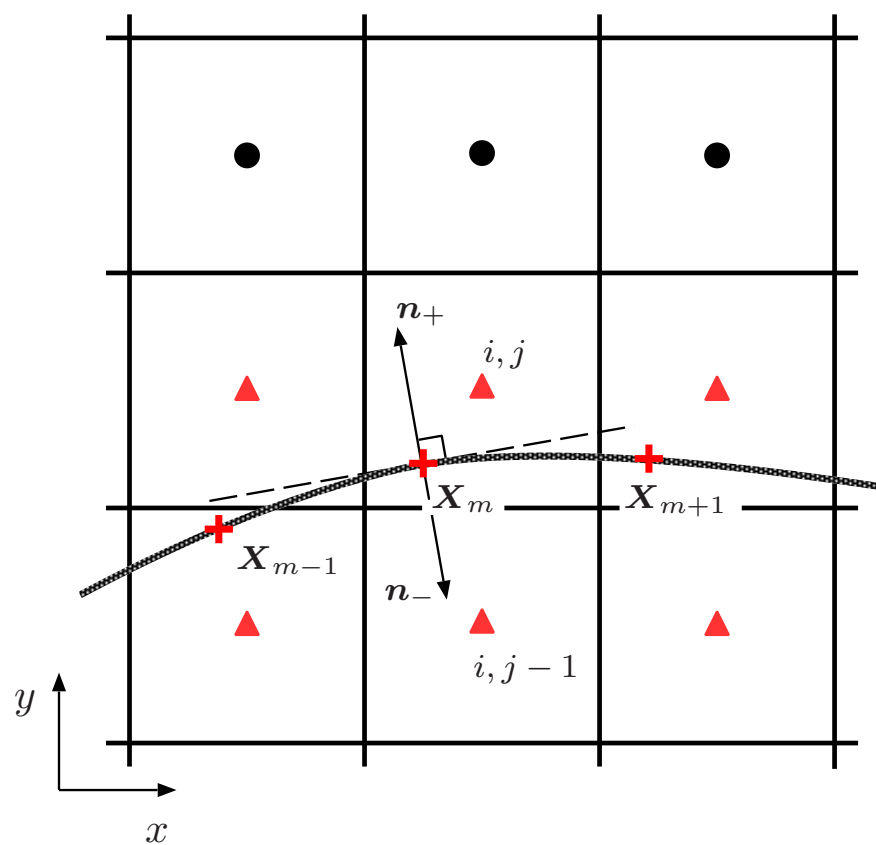

Figure 4: Schematic of the method for calculating the fluid stress defined on Lagrangian marker. The membrane location is indicated by a thick line, and the Lagrangian marker is represented by the plus symbol. 


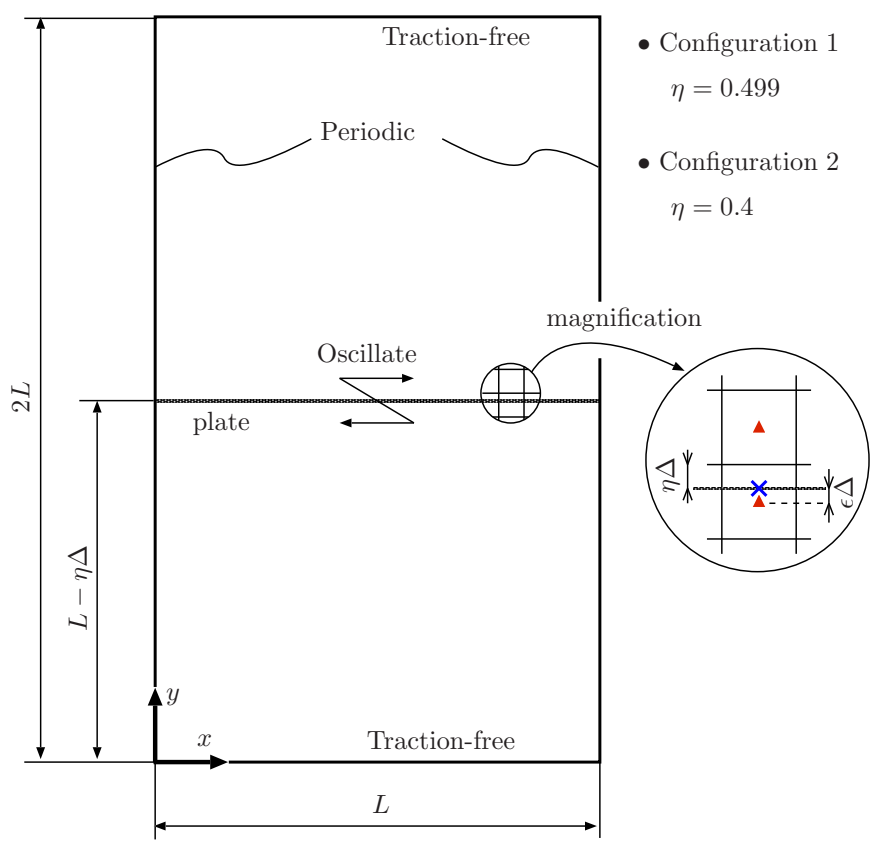

Figure 5: Schematic of the flow around an oscillating plate.

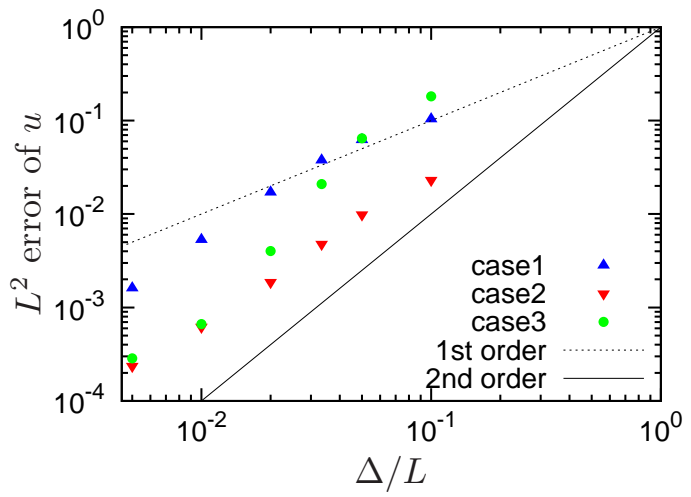

(a) $L^{2}$-norm errors

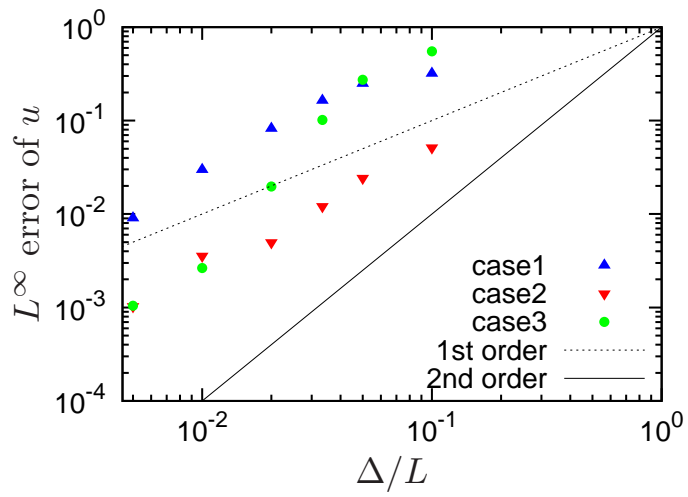

(b) $L^{\infty}$-norm errors

Figure 6: The errors in the $x$-velocity of the fluid induced by the oscillating plate of Configuration $1(\delta=$ $0.499 \Delta$ ) plotted against the spatial resolution. 


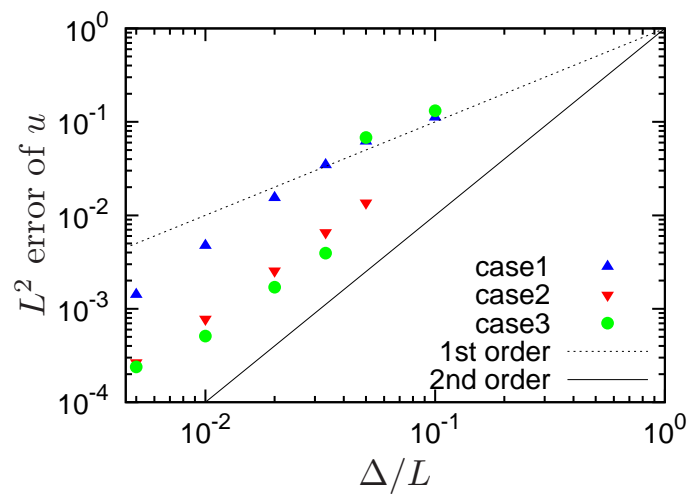

(a) $L^{2}$-norm errors

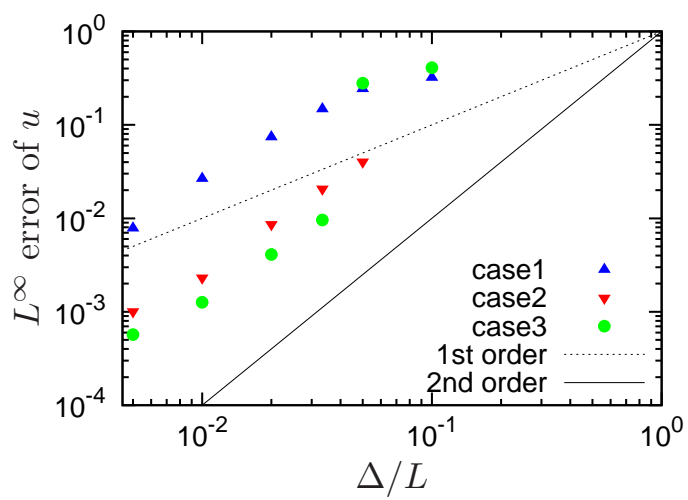

(b) $L^{\infty}$-norm errors

Figure 7: The errors in the $x$-velocity of the fluid induced by the oscillating plate of Configuration $2(\delta=$ $0.4 \Delta$ ) plotted against the spatial resolution.

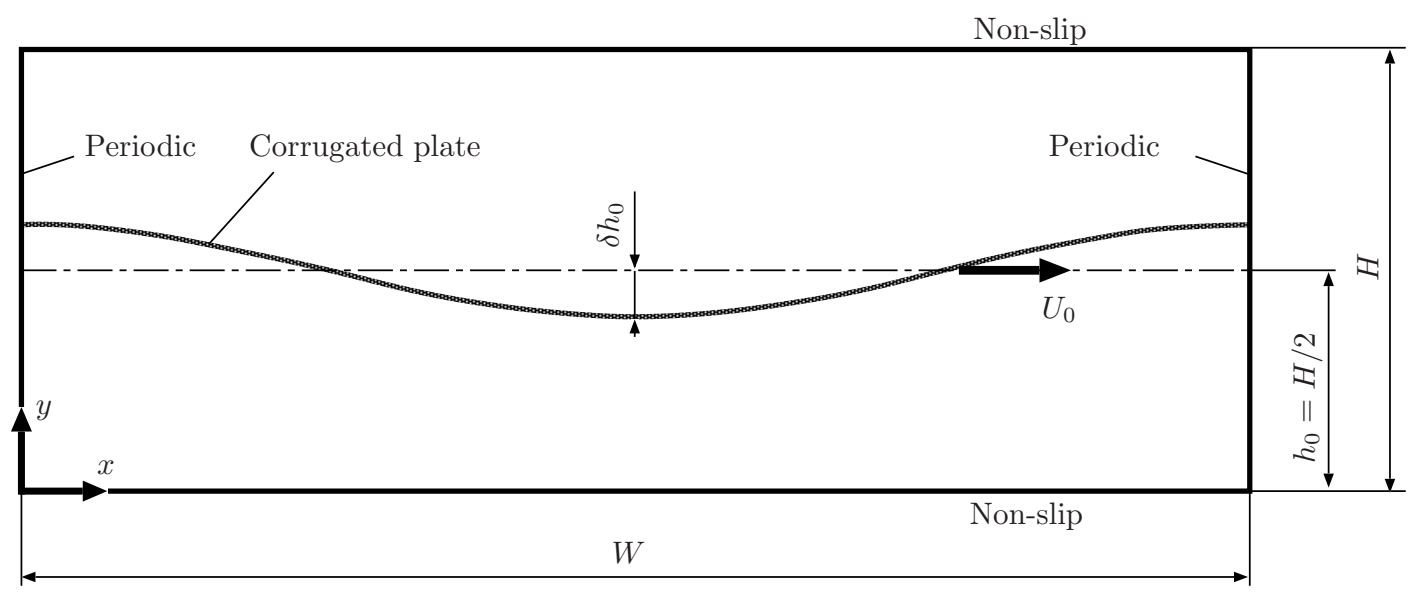

Figure 8: Schematic of a moving corrugated plate in a parallel channel. The plate is a sinusoidal geometry, and it moves at a constant speed in the $+x$ direction.

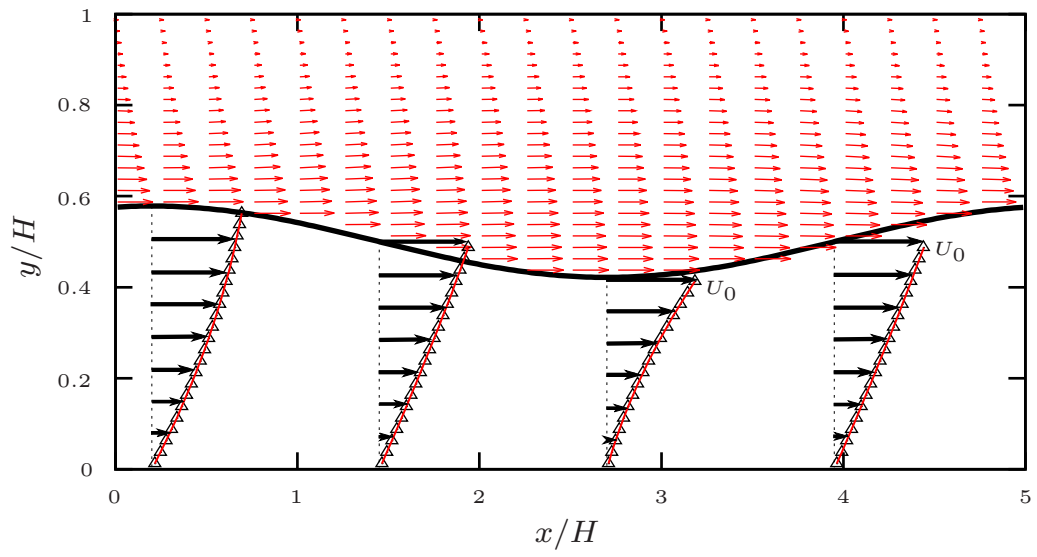

Figure 9: Instantaneous flow field driven by the corrugated plate after reaching steady state. Upper region of the corrugated plate is the velocity vector $(u, v)$. Lower region compares the streamwise $(x)$ velocity profiles with the analytical solution based on the lubrication assumption. Different velocity scales are applied for the upper and lower plots. 


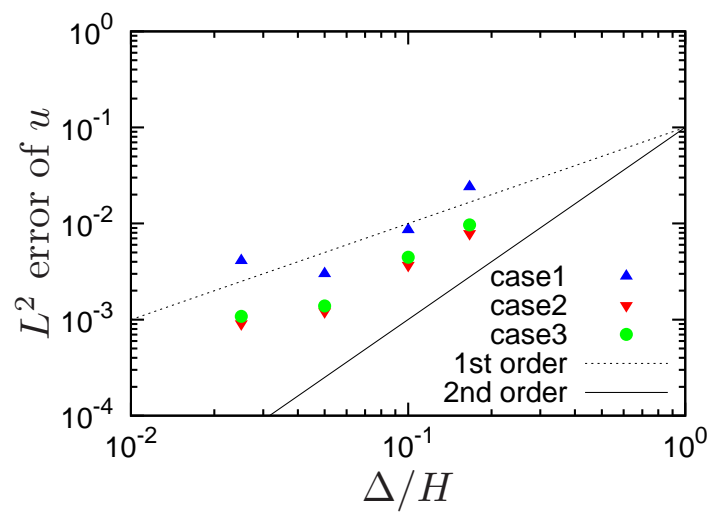

(a) $L^{2}$-norm errors

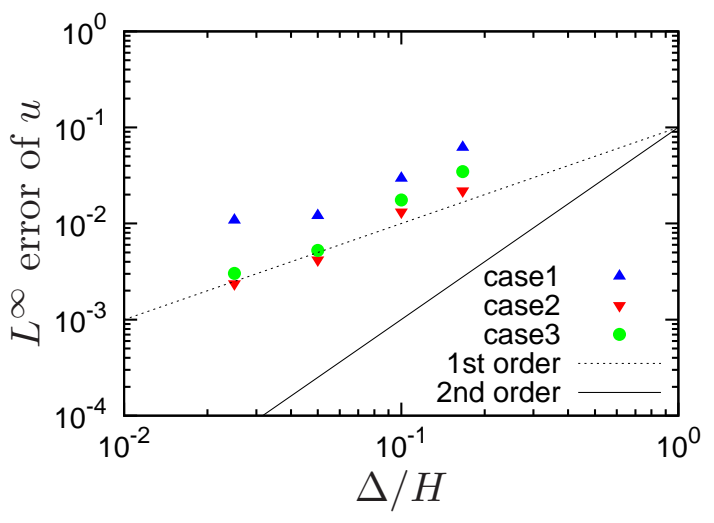

(b) $L^{\infty}$-norm errors

Figure 10: The errors in the flow velocity induced by the moving corrugated plate plotted as a function of the spatial resolution.

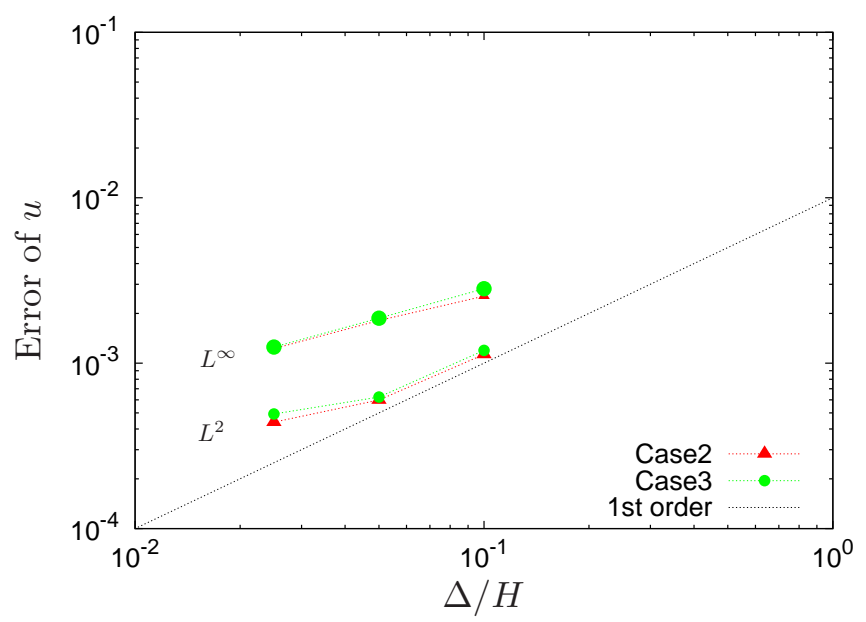

Figure 11: The errors with respect to the approximate analytical solution Eq.(39) when the amplitude of the undulating geometry $\delta$ is decreased to 0.1 (with keeping $\operatorname{Re}=1.5$ ). 


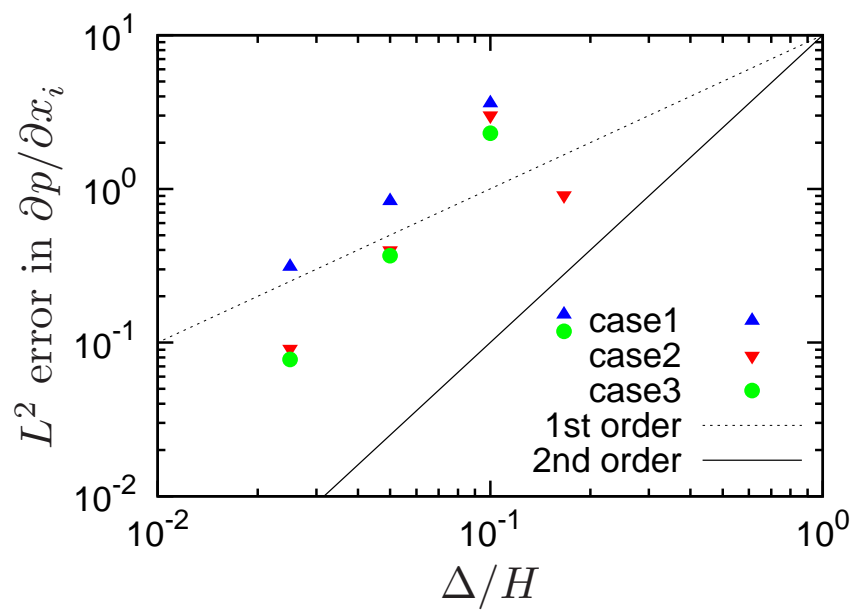

Figure 12: The errors in the pressure gradient as a function of the spatial resolution.

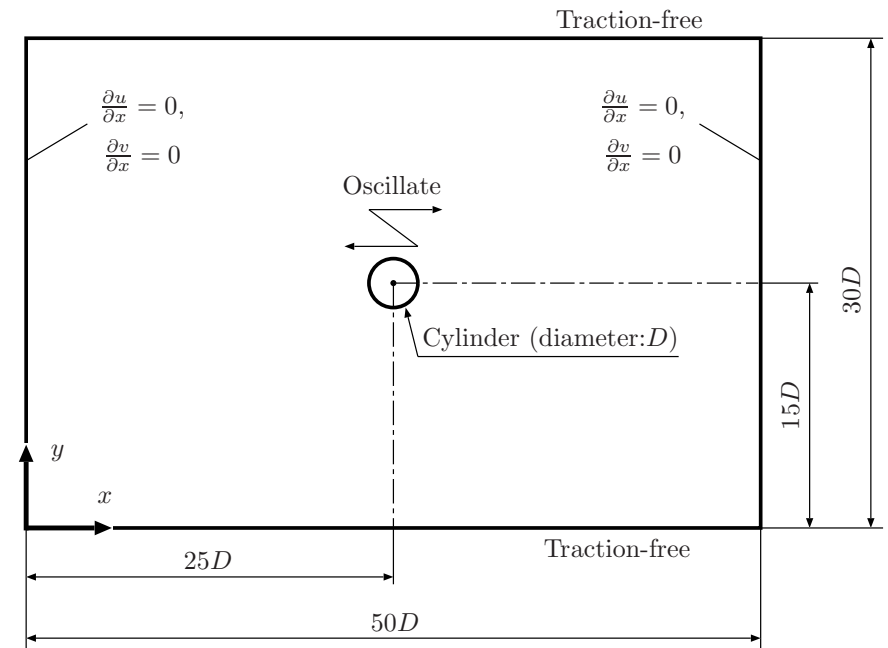

Figure 13: Schematic of the flow field around an oscillating circular cylinder. 


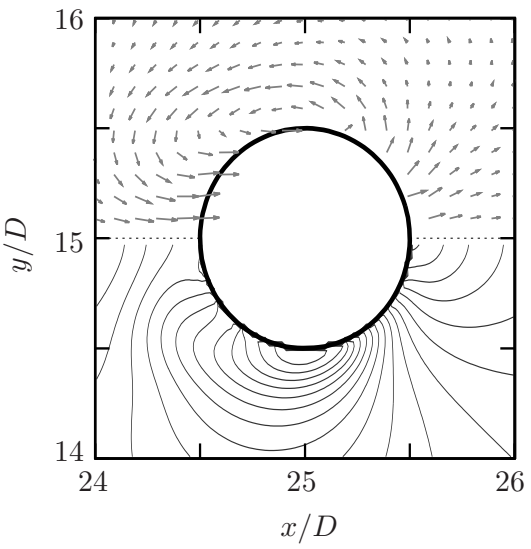

(a)

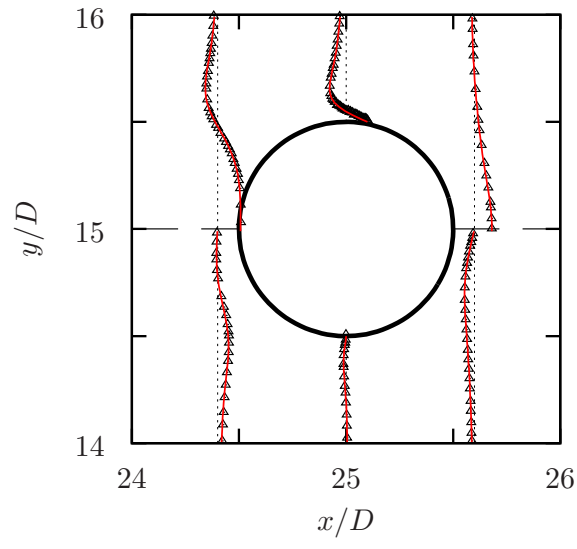

(b)

Figure 14: Snapshots of the flow field (under $\mathrm{Re}=100$ and $\mathrm{KC}=5$ ) at the oscillation phase of $\pi$. (a) The upper-half side shows the velocity field and the lower-half side is the pressure field. The vectors are plotted at every five grid points, and the pressure contours are drawn within the value range between -1 and 1 at the intervals of 0.1 . (b) The velocity profiles at three cross-sections $(0.1 D$ up- and down-stream sides of the leading and trailing edges and the mid cross-section) and a comparison with [19]. The upperand lower-half sides are the profiles of $u$ and $v$, respectively. The lines represent the result by Case 2 and the symbols are the results of Dütsch et al. [19].

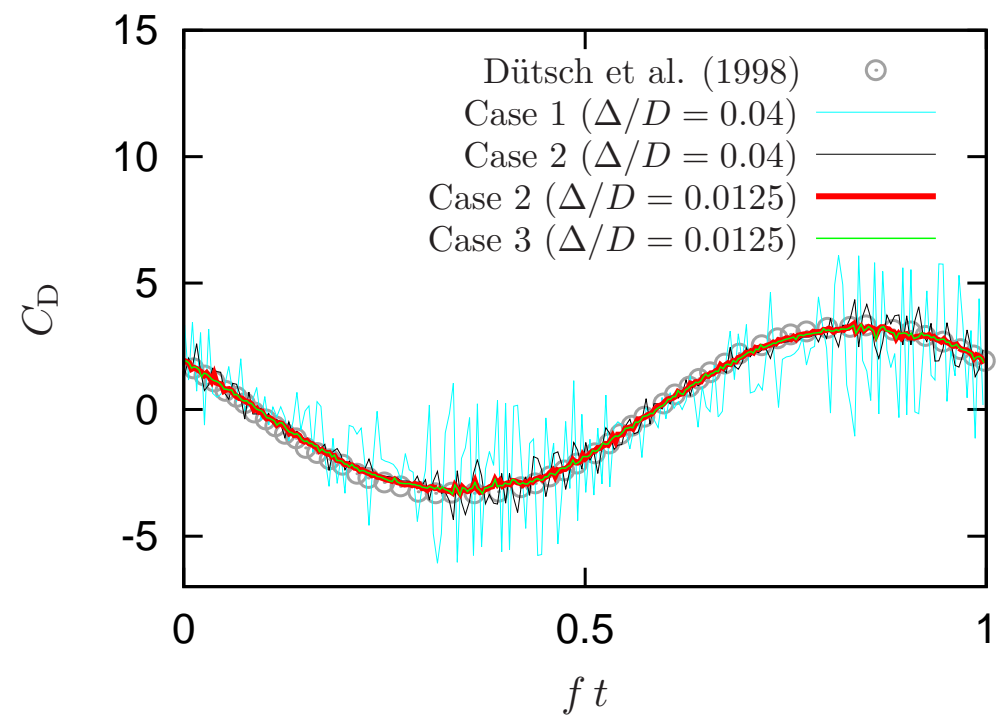

Figure 15: The time histories of the drag coefficients for different spatial resolutions: $\Delta / D=0.04$ for Cases 1 and 2 , and $\Delta / D=0.0125$ for Cases 2 and 3. The lines are the computational results and symbol is the result of Dütsch et al. [19]. 


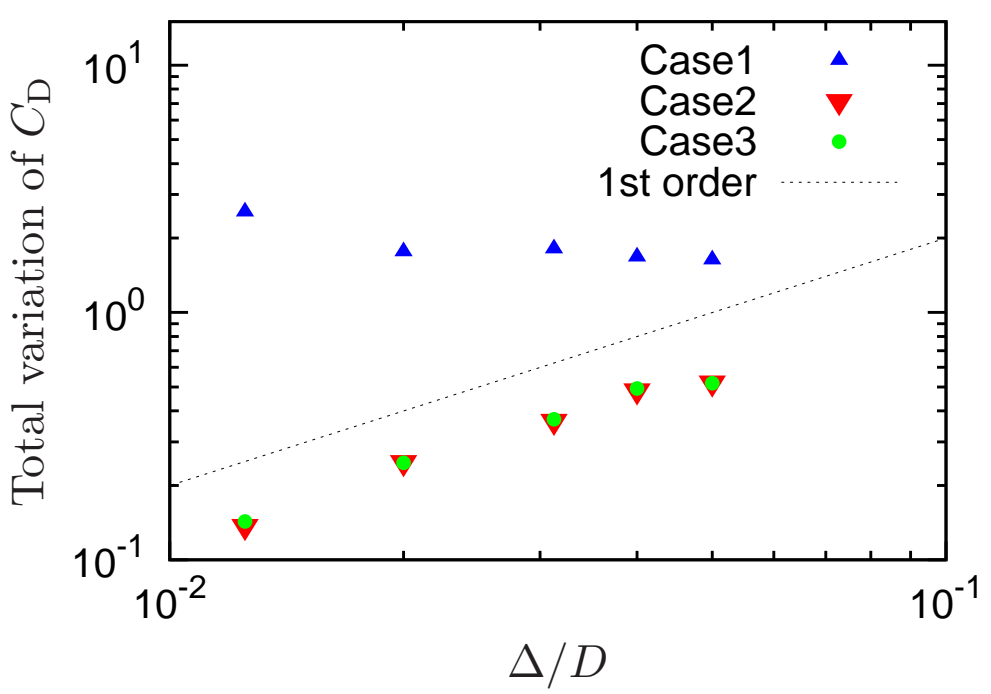

Figure 16: Total variation of the error in the drag coefficient plotted against the spatial resolution.

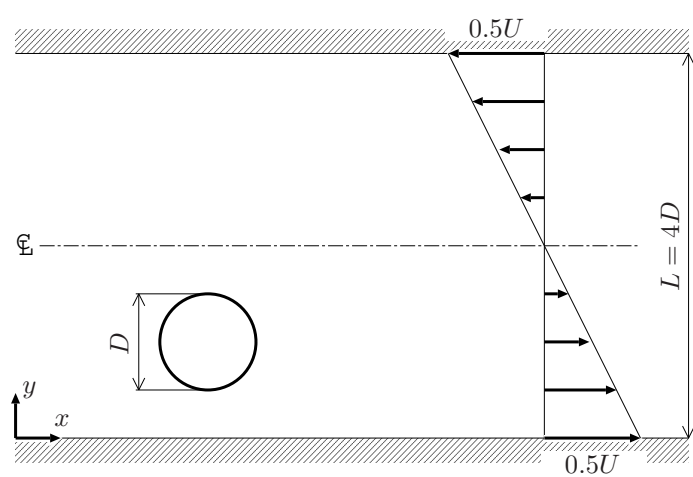

(a) Schematic

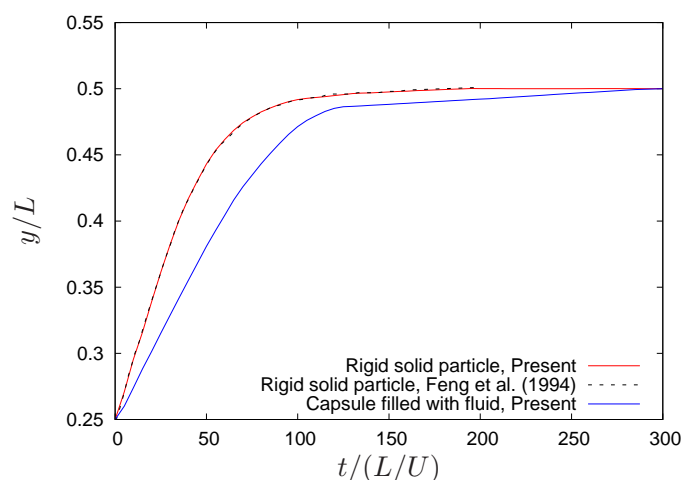

(b) Time history

Figure 17: Lateral migration of a neutrally buoyant particle in a simple shear flow with no gravity. The bulk Reynolds number is $U L / \nu=40$ and the particle Reynolds number is $U(D / 2)^{2} / \nu L=0.625$. The initial position of the particle centre is $y=D$, and released into the flow with no translating and angular velocities. 


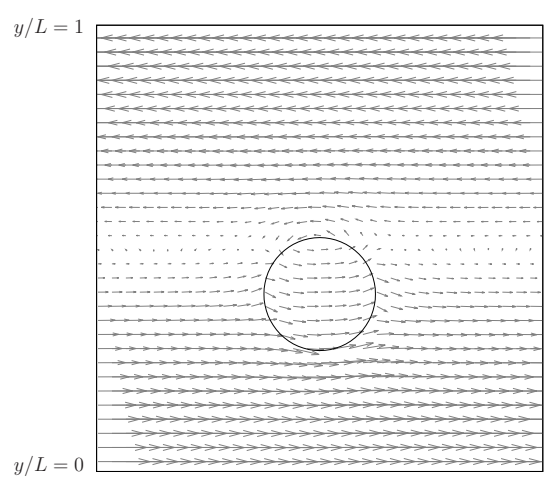

(a) $t=60$

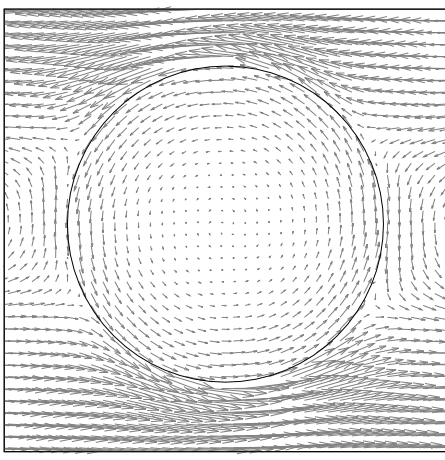

(b) $t=60$ (on trans. frame)

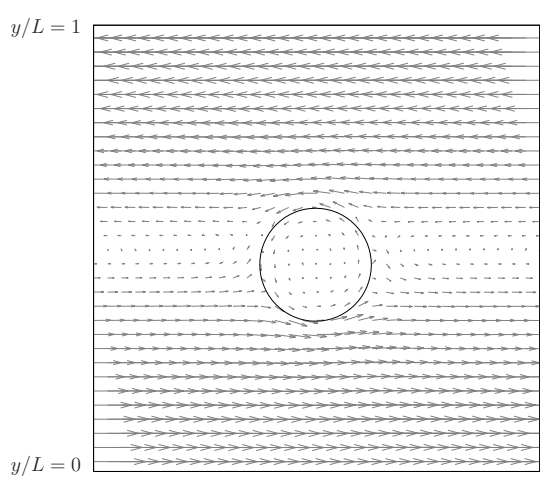

(c) $t=135$

Figure 18: Velocity vectors inside and outside the capsule. (a) and (c) are different time instances and the arrows are plotted at every 10 grid points in both directions. (b) is the same instance as (a), but (b) is observed on the moving frame of reference at the particle translating velocity, and the arrows are plotted at every 3 grid points.

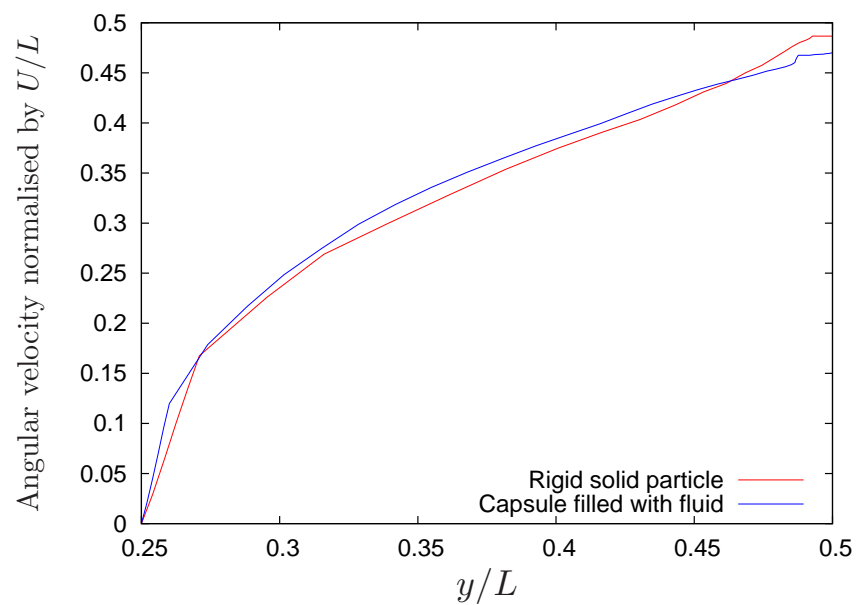

Figure 19: The angular velocity as a function of the wall-normal distance.

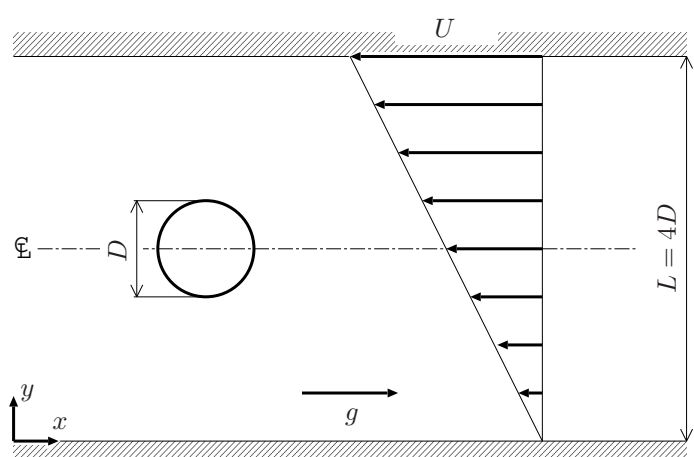

(a) Schematic

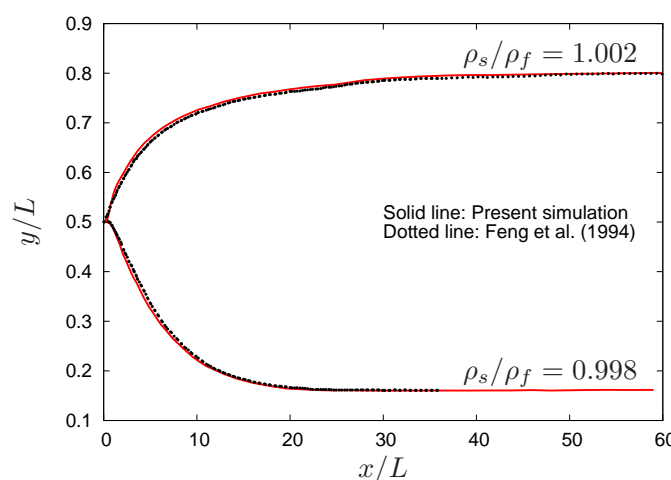

(b) Trajectory

Figure 20: Lateral migration of a non-neutrally buoyant particle in a simple shear flow under the effect of gravity. The bulk Reynolds number is $U L / \nu=40$ and the Froude number is $g L / U^{2}=392$. 


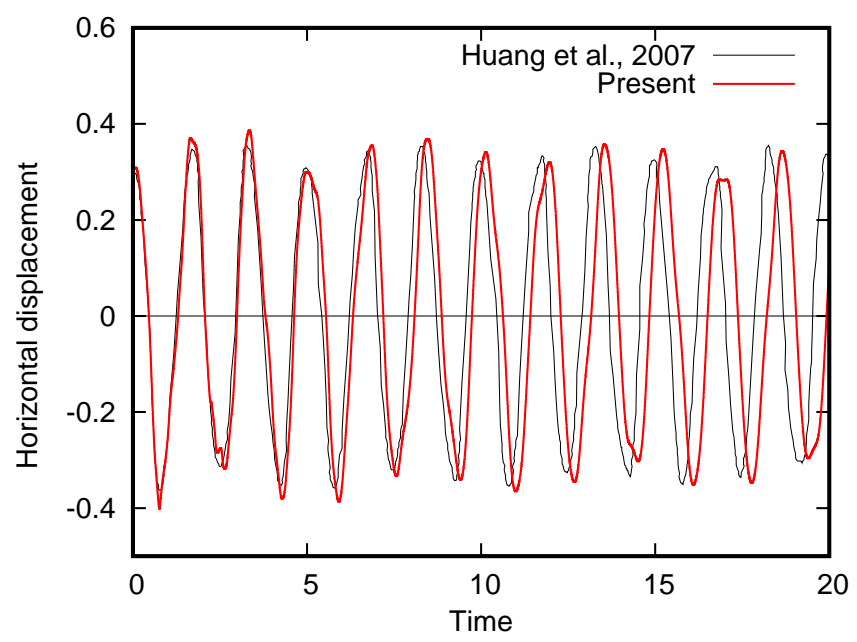

Figure 21: Comparison of the time evolution of the motion of the filament under the influence of gravity (with no effect of the ambient fluid). One end of the filament is free and the other end is fixed in space with the simply-supported condition. The time history of the horizontal displacement of the free end is plotted.

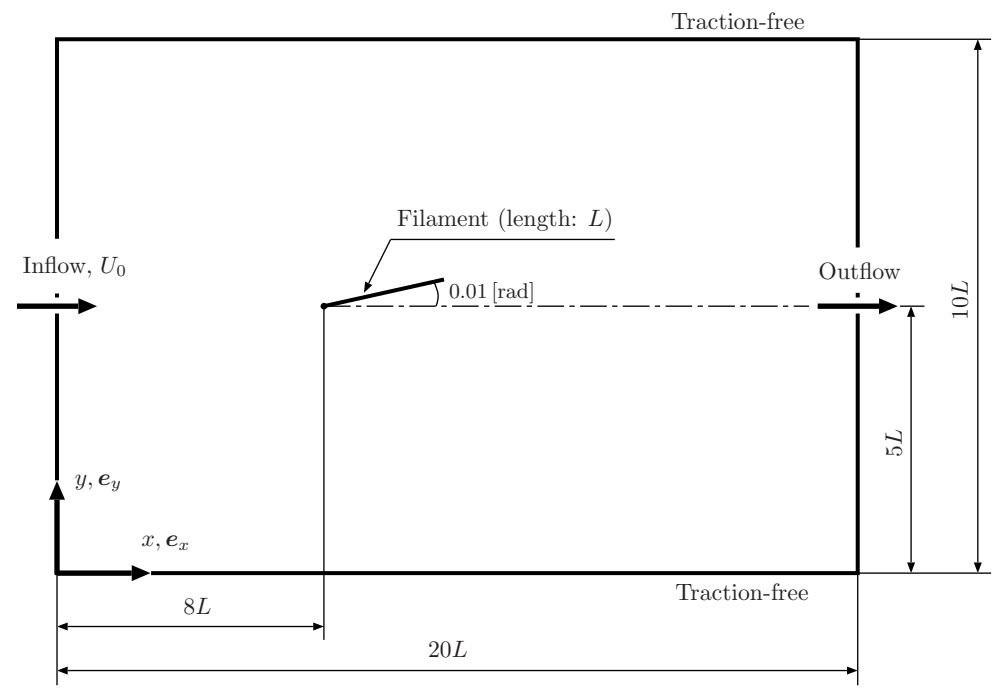

Figure 22: Schematic of a 2-D filament in a uniform stream. The filament is initially stretched and tilted by $0.01[\mathrm{rad}]$ against the $x$ axis. Throughout the simulation, the leading edge of the filament is pinned and the trailing edge is free. 


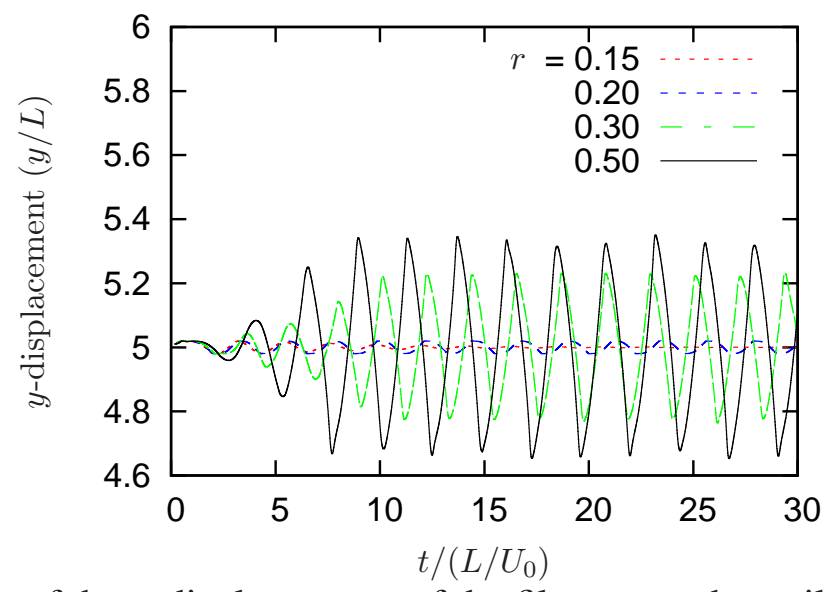

Figure 23: The time histories of the $y$-displacements of the filament at the trailing edge for different density ratios $r\left(=\rho_{\mathrm{s}} h /\left(\rho_{\mathrm{f}} L\right)\right)$.

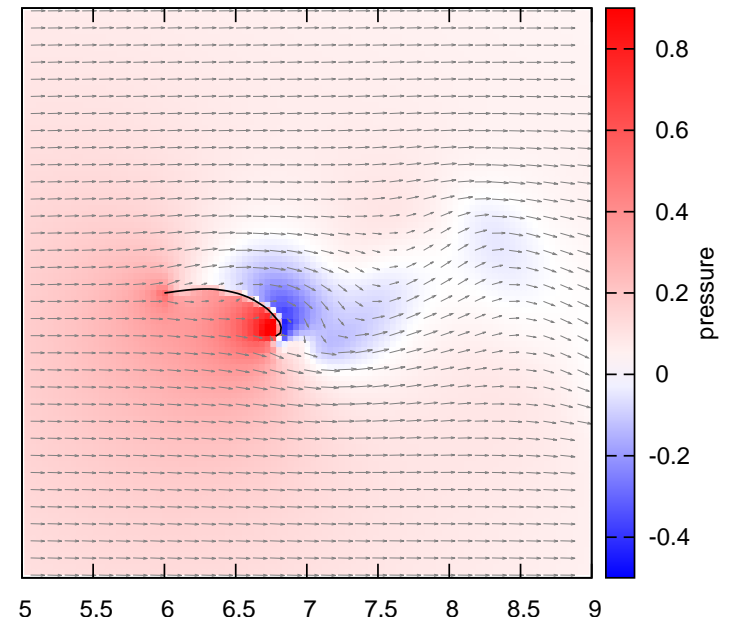

(a) $t=20.0 \mathrm{~L} / U_{0}$

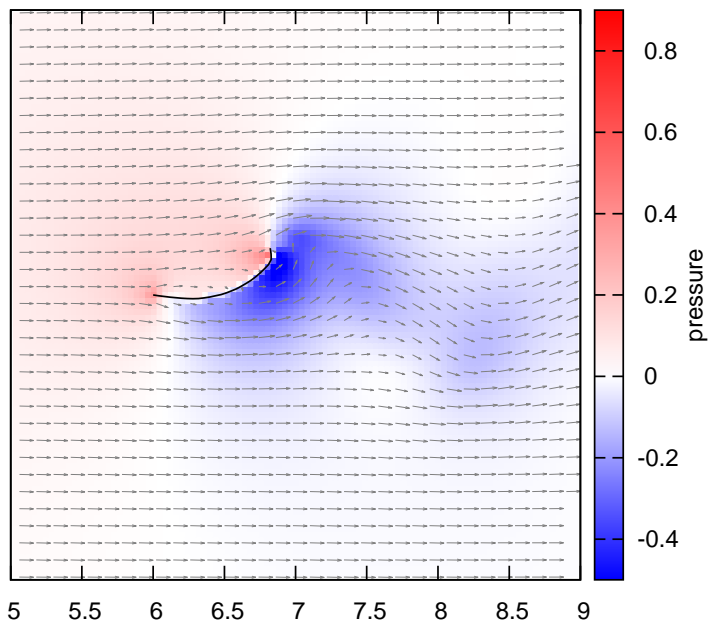

(b) $t=21.2 \mathrm{~L} / U_{0}$

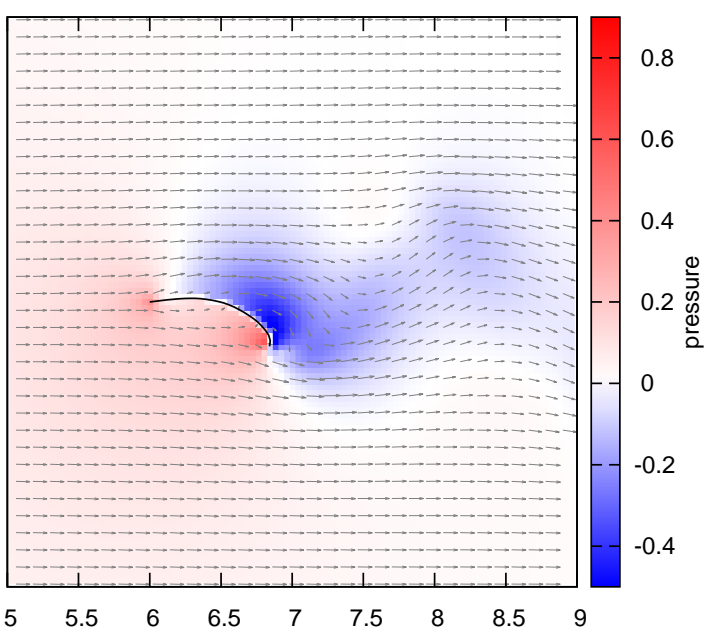

(c) $t=22.4 L / U_{0}$

Figure 24: Sequential snapshots of the velocity and pressure fields around a flapping filament $(r=0.5)$ at the intervals of $1.2 L / U_{0}$. The pressure is non-dimensionalised by $\rho_{f} U_{0}^{2}$. 


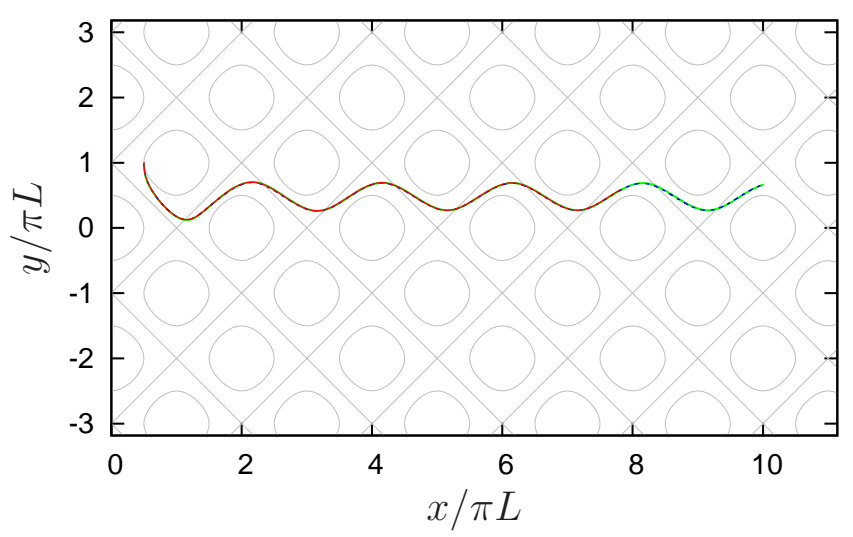

Figure 25: The trajectory of a rigid spherical particle in the Taylor-Green vortices. The density ratio of the the particle to the ambient fluid is 10 , and gravity is not imposed. The coloured lines compare the trajectory of the particle by the present method (red solid line) with those obtained by the different methods in Ref. [21]: blue dashed line, fully-resolved simulation by an immersed boundary method [9]; green solid line, a simulation based on a volume-averaged equation considering the surface stress and flow disturbance [21]. The grey line shows the streamlines of the undisturbed flow of the Taylor-Green vortices. 